\title{
Targeted Delivery of Adamantylated Peptidoglycan Immunomodulators in Lipid Nanocarriers: NMR Shows That Cargo Fragments Are Available on the Surface
}

\author{
Rosana Ribić, Mateja Manček-Keber, Fernando Chain, Davy Sinnaeve, José C. Martins, Roman Jerala, \\ Srđanka Tomić, and Krisztina Fehér*
}

Cite This: https://dx.doi.org/10.1021/acs.jpcb.0c00029

Read Online

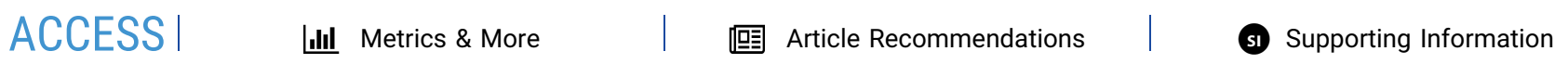

ABSTRACT: We present an in-depth investigation of the membrane interactions of peptidoglycan (PGN)-based immune adjuvants designed for lipid-based delivery systems using NMR spectroscopy. The derivatives contain a cargo peptidoglycan (PGN) dipeptide fragment and an adamantyl group, which serves as an anchor to the lipid bilayer. Furthermore, derivatives with a mannose group that can actively target cell surface receptors on immune cells are also studied. We showed that the targeting mannose group and the cargo PGN fragment are both available on the lipid bilayer surface, thereby enabling interactions with cognate receptors. We found that the nonmannosylated compounds are incorporated stronger into the lipid assemblies than the mannosylated ones, but the latter compounds penetrate deeper in the bilayer. This might be explained by stronger electrostatic interactions available for zwitterionic nonmannosylated derivatives as opposed to the compounds in which

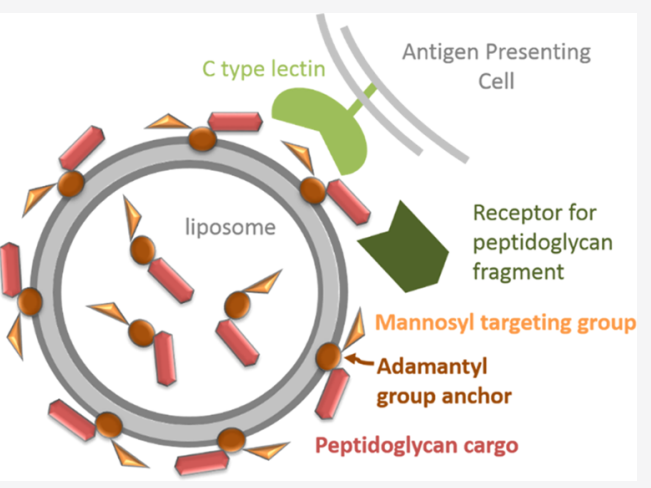
the charged N-terminus is capped by mannose groups. The higher incorporation efficiency of the nonmannosylated compounds correlated with a larger relative enhancement in immune stimulation activities upon lipid incorporation compared to that of the derivatives with the mannose group. The chirality of the adamantyl group also influenced the incorporation efficiency, which in turn correlated with membrane-associated conformations that affect possible intermolecular interactions with lipid molecules. These findings will help in improving the development of PGN-based immune adjuvants suitable for delivery in lipid nanoparticles.

\section{INTRODUCTION}

Development of safe and effective adjuvants in vaccine research is still a challenge ${ }^{1}$ as many effective immune modulatory compounds have significant adverse effects, while commonly used safe adjuvants, such as alum, ${ }^{2}$ are not very potent. Stimulation of the immune response and modulation of the appropriate type of immunity can be achieved by activation of the innate immune system via activation of pattern recognition receptors (PRRs) expressed on immune cells and other cells. Targeting different PRRs by microbial danger signals, so-called pathogen-associated molecular patterns (PAMPs), triggers signaling cascades, leading to specific proinflammatory responses. Immune adjuvants with various characteristics thus can be obtained using fragments or analogues of PAMPs. ${ }^{3}$

Bacterial cell wall peptidoglycan (PGN) is one of the most important microbial signatures recognized by innate immune receptors. PGN is recognized ${ }^{4}$ by intracellular nucleotide oligomerization domain (NOD)-like receptors NOD1 and NOD2 and secreted peptidoglycan recognition proteins, among others. It has been shown that polymeric PGN can stimulate immune defenses even without the presence of an infection; therefore, it can act as a very potent adjuvant. ${ }^{5}$
However, it also has too many side effects to be used in vaccines. ${ }^{6}$ Fragments and substances ${ }^{7}$ derived from PGN also exhibit weaker adjuvant activities with diminished adverse effects. One of the most widely used PGN fragments is muramyl dipeptide ${ }^{8}$ (MDP) containing the $N$-acetylmuramylL-alanyl-D-isoglutamine (L-Ala-D-iGln) motif of PGN, which is the minimal essential unit of PGN. MDP is recognized by NOD 2,9 a cytoplasmic receptor of the innate immune system, and has a weak immune modulatory effect ${ }^{8}$ as it is rapidly excreted into the urine, and its administration is associated with various adverse side effects.

To improve the adjuvant properties of PGN fragments, an appropriate drug delivery system is needed, which can improve metabolic stability and bioavailability, enable reduction of therapeutic doses, and thereby reduce adverse effects. The use

Received: January 6, 2020

Revised: March 20, 2020

Published: April 14, 2020 
Scheme 1. Chemical Structure of the Investigated PGN Derivatives ${ }^{a}$

(a) D-Ad 1 Tp DAda-LAla-DiGIn<smiles>[Y4][C@H](NC(=O)[C@@H](N)C12CC3CC(CC(C3)C1)C2)C(=O)N[C@@H](CCC(=O)O)C(N)=O</smiles>

(b) L- $-\mathrm{Ad}_{1} \mathrm{Tp}$

LAda - LAla - DiGIn<smiles>C[C@H](NC(=O)[C@@H](N)C12CC3CC(CC(C3)C1)C2)C(=O)N[C@@H](CCC(=O)O)C(N)=O</smiles>

(c) Man-D-Ad 1 Tp Man - R-Lnk - DAda - LAla-DiGln<smiles>C[C@@H](COC(O)C1C(O)C(O)C(CO)C1(O)CO)C(=O)N[C@H](C(=O)N[C@@H](C)C(=O)N[C@@H](CCC(=O)O)C(N)=O)C12CC3CC(CC(C3)C1)C2</smiles>

(d) Man-L-Ad 1 Tp Man - R-Lnk - LAda - LAla - DiGIn

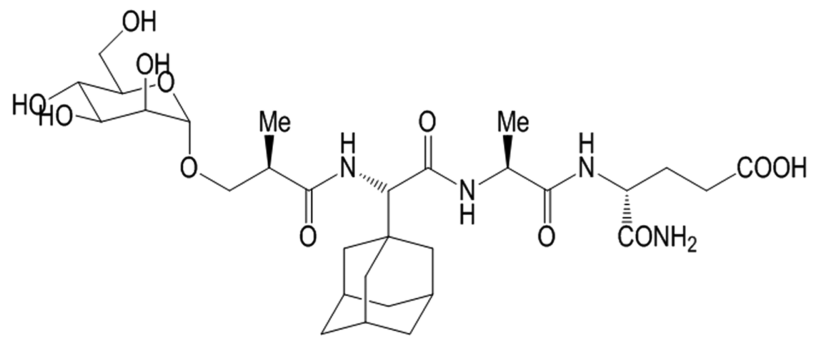

$a_{\text {D-Ad }}$ Tp: D-(adamant-1-yl)-Gly-L-Ala-D-isoGln, L-Ad 1 Tp: L-(adamant-1-yl)-Gly-L-Ala-D-isoGln, Man-D-Ad 1 Tp: $(2 R)-N$-[3- $(\alpha$-D-mannopyranosyloxy)-2-methylpropanoyl]-D-(adamant-1-yl)-Gly-L-Ala-D-isoGln, and Man-L-Ad 1 Tp: (2R)- $N$-[3-( $\alpha$-D-Mannopyranosyloxy)-2-methylpropanoyl]-L(adamant-1-yl)-Gly-L-Ala-D-isoGln. The "Ad," notation indicates an adamant-1-yl attachment, and the "Tp" notation stands for a tripeptide. Ada stands for adamantylated glycine, D-iGln for D-isoglutamine, and Lnk for the linker residue including the $-\mathrm{CH}_{2}-\mathrm{CH}(\mathrm{Me})-\mathrm{fragment}$.

of lipid nanoparticles as carriers is well-established ${ }^{10}$ and has found widespread applications in vaccination as many carriers are able to stimulate the immune system and induce both cellular and humoral immune responses. ${ }^{11}$ Similarly, many lipophilic derivatives of MDP were developed to increase loading in lipid carriers, ${ }^{11,12}$ which increased adjuvant activities and reduced side effects. ${ }^{13}$

The PGN derivatives ${ }^{14}$ we investigate in this study are designed to be suitable for encapsulation into liposomes. These derivatives contain only a minimal PGN fragment, L-Ala-D-iGln payload, and an adamantyl group to increase hydrophobicity and to facilitate anchoring of the PGN fragment cargo to the membrane. Due to the chirality of the adamantyl group, two diastereoisomers were obtained (Scheme 1a,b), which were both shown to be stable, nonpyrogenic, water-soluble, and nontoxic and displayed an adjuvant effect in vivo in mice models. $^{15,16}$

Adamantane-based drugs are used as antivirals ${ }^{17,18}$ as well as in the treatment of Parkinson's ${ }^{19,20}$ and Alzheimer's ${ }^{21,22}$ diseases. They often act as channel blockers, ${ }^{23-25}$ but their therapeutic effect was also suggested to be related to nonspecific interactions with membranes. ${ }^{26,27}$ Indeed, the hydrophobicity and unique geometry of adamantane are thought to increase the membrane permeability and adsorption of drugs and as a result significantly modify the pharmacological profile of drug molecules. Amantadine (1-aminoadamantane) is one of the oldest and most well-known adamantane-containing drugs, and its membrane interaction is thought to play a crucial role in its activity. ${ }^{28-30}$

We also investigated versions of the adamantylated PGN compounds conjugated to mannose moieties (Scheme 1c,d). Glycosylation with carbohydrates that specifically bind to endogenous lectin receptors on cell surfaces achieves active targeting, which is an important aspect of any drug delivery system as it is more controllable and efficient than passive targeting. Effectiveness of targeting using mannose was shown for mannosylated liposomes, which were found to enhance the uptake and activation of dendritic cells and to increase $\mathrm{T}$ cell proliferation. ${ }^{31}$ In this fashion, antigen-presenting cells (APCs) are targeted, such as macrophages and dendritic cells that express C-type lectins (CLRs), which can recognize the mannose-targeting group. CLR recognition may result in improved internalization and presentation of antigens by antigen-presenting cells ${ }^{32}$ and may also modulate the immune 
response via signaling pathways. ${ }^{33}$ The former could help in increasing the uptake of the mannosylated compounds and thus achieve active targeting, while the latter might lead to the modification of the host defense response triggered by the PGN fragment via PRR crosstalk. The adjuvant activity of the mannosylation in the adamantylated compounds was indeed found to be amplified in vivo, ${ }^{14,16}$ which might be related to the successful targeting of immune cells.

The PGN derivatives exhibited adjuvant activities in vivo in experiments performed in mice models using ovalbumin (OVA) as an antigen. ${ }^{14,15}$ Differences regarding the configuration on the stereogenic center of adamantyl glycine were observed as D-Ad $\mathrm{Ap}_{1} \mathrm{p}$ showed higher stimulation of anti-OVA immunoglobulin $\mathrm{G}$ ( $\mathrm{IgG}$ ) antibody production in comparison to $\mathrm{L}-\mathrm{Ad}_{1} \mathrm{Tp} .{ }^{15}$ The best adjuvant activity was observed for the mannosylated derivate Man-D-Ad ${ }_{1} \mathrm{Tp}^{16}$ Adjuvant effects of the adamantylated PGN derivatives were also assessed in an in vitro assay ${ }^{34}$ monitoring cytokine and chemokine production in immortalized mouse bone marrow-derived macrophages. We found that while the PGN compounds do not stimulate macrophages alone, $\mathrm{L}-\mathrm{Ad}_{1} \mathrm{Tp}$ and, in particular, Man-L-Ad $\mathrm{T} \mathrm{p}$ were able to enhance the immune responses induced by lipopolysaccharide (LPS) on macrophages. Moreover, the costimulation activity on macrophages was further increased by the incorporation of the compounds into phosphatidylcholine liposomes.

To develop improved adjuvant delivery systems for vaccination, understanding of the molecular details governing the encapsulation of adjuvants into lipid bilayers and their interactions with the targeted receptors is mandatory. NMR spectroscopy is a powerful technique for investigating the molecular details of membrane interactions of lipid-embedded molecules such as detection of binding, ${ }^{35}$ membraneassociated structures of solutes, ${ }^{36}$ their position and orientation in the membrane, ${ }^{30,37}$ and determination of entrapment efficiency. ${ }^{38}$ The sign dependence ${ }^{35}$ of the nuclear Overhauser effect (NOE) on the size of the molecule associated with the membrane can reveal the presence of a weak interaction, ${ }^{36}$ and the structure of the membrane-bound conformation can be determined using intramolecular transferred NOEs. Diffusion ordered spectroscopy (DOSY) ${ }^{39}$ provides means to measure the lipid entrapment efficiency of molecules ${ }^{38}$ by determining the ratio of the free and bound solute in the case of fast exchange between the solutes and the bilayer. Furthermore, saturation transfer difference (STD) ${ }^{40}$ spectroscopy is used to probe the depth and orientation of the molecule within the lipid bilayer ${ }^{30}$ via an epitope mapping procedure. ${ }^{41,42}$ Solventaccessible fragments of molecules immersed in membranes can be monitored ${ }^{37}$ using water-soluble paramagnetic probes by inducing paramagnetic relaxation enhancement (PRE), ${ }^{43}$ which can be detected by the measurement of transverse relaxation times ${ }^{44}\left(T_{2}\right)$.

To promote the therapeutic impact of the antigen and adjuvant payload, vaccine formulations are required ${ }^{45}$ to retain their cargo and to improve their exposure to receptors. In this report, we investigated all four PGN fragment-containing derivatives shown in Scheme 1 using the above NMR spectroscopic methods to reveal the molecular details of their interaction with lipid molecules such as their lipid encapsulation efficiency, their membrane-associated conformations, and the location and orientation of the derivatives within the bilayer. These investigations allow assessing whether sufficient membrane incorporation can be achieved for their safe delivery in a lipid-based formulation and also to reveal the availability of the targeting group and the PGN cargo fragment toward interaction with their corresponding receptors for active targeting.

Since NMR investigation of large liposomes is not straightforward due to their size and heterogeneity, we used isotropic bicelles ${ }^{46,47}$ as model bilayer systems. Here, a shortchain phospholipid, used as a detergent, is mixed with longchain phospholipids, thereby forming disklike bilayers, ${ }^{48-50}$ with the rims covered by the detergent-like short-chained phospholipid molecules. Bicelles are considered as good models of lipid assemblies with bilayer structures. ${ }^{3-53}$ Specifically, phosphatidylcholine lipids with dimyristoyl and dihexanoyl chains were used as model lipids (1,2-dimyristoylsn-glycero-3-phosphocholine (DMPC) $)^{54}$ and detergents $(1,2-$ dihexanoyl-sn-glycero-3-phosphocholine (DHPC)), respectively. Both DMPC and DHPC carry positively charged choline groups and negatively charged phosphate groups in the head region, which is then bridged by polar glycerol moieties to the fatty acid tails. For the purpose of the NMR characterization, we utilized a molar ratio of 0.5 between the detergent and bilayer-forming lipids to generate isotropically tumbling bicelles.

\section{EXPERIMENTAL AND COMPUTATIONAL METHODS}

Materials. Protonated 1,2-dimyristoyl-sn-glycero-3-phosphocholine (DMPC) and 1,2-dihexanoyl-sn-glycero-3-phosphocholine (DHPC) with more than 99\% purity were purchased from Avanti Polar Lipids, Inc. in a powder form. Deuterium oxide $\left(\mathrm{D}_{2} \mathrm{O}\right)$ with a purity of more than 99.9 atom $\% \mathrm{D}$ was purchased from EurisoTop (France). N-Acetylglucosamine (NAG) was purchased from Sigma-Aldrich. PGN derivatives were synthesized as previously described. ${ }^{14,15}$

NMR Spectroscopy. The NMR samples contained ca. 20 $\mathrm{mg}$ of the PGN derivatives dissolved in $500 \mu \mathrm{L}$ of water containing $10 \% \mathrm{D}_{2} \mathrm{O}$. Later, an aliquot of the aqueous sample was used for preparing a lipid bicelle sample with ca. $8 \mathrm{mM}$ PGN compound concentration in $100 \mathrm{mM} \mathrm{K}$ phosphate buffer at $\mathrm{pH}$ 7.2. The concentrations of $100 \mathrm{mM}$ DMPC and 200 $\mathrm{mM}$ DHPC were used for making the lipid bicelle solution with a molar ratio of $q=0.5$ according to described procedures. ${ }^{47}$ NMR experiments were performed at 298.3 and $310.2 \mathrm{~K}$ on a Bruker Avance II NMR spectrometer operating at $700.13 \mathrm{MHz}$ equipped with a $5 \mathrm{~mm}$ TXI-Z probe. The ${ }^{1} \mathrm{H}$ resonances were assigned using standard homonuclear and heteronuclear correlation experiments.

Determination of Diffusion Coefficients. Diffusion coefficients were measured by pulsed-gradient stimulatedecho (PGSTE) NMR with a stimulated spin echo sequence with bipolar gradients, one spoil gradient, and a 3-9-19 water suppression scheme. ${ }^{55}$ The maximum gradient strength of the probe was $57.7 \mathrm{G} / \mathrm{cm}$. The diffusion delay was set to $200 \mathrm{~ms}$, while the gradient length was $2 \mathrm{~ms}$ and varied in intensity between 2 and $95 \%$ of the maximum gradient strength in 64 steps. Spectra were recorded with 256 scans. Two-dimensional (2D) DOSY plots were generated using TOPSPIN 3.1. Nonoverlapping resonances and showing pure monoexponential decay were used to extract the diffusion coefficient of the associated species by fitting to the appropriate StejskalTanner equation ${ }^{56}$ using an in-house fitting procedure implemented in MATLAB. 
Structure Calculation of the Membrane-Associated

State. Distance restraints were extracted from a 2D NOE spectroscopy (NOESY) spectrum recorded with $100 \mathrm{~ms}$ mixing time at $700.13 \mathrm{MHz}$ at room temperature. All transferred NOE cross-peaks were manually assigned and integrated using the CCPNMR software. ${ }^{57}$ Signals arising from $\gamma$ and $\varepsilon$ positions of methylene groups in the adamantyl moiety could not be unambiguously assigned; therefore, ambiguous restraints were implemented. The signal intensities were converted into distances by calibration against the average of the integrals of all NOE intensities, corresponding to a reference distance of $3.2 \AA$. For structure calculations, the simulation engine CNS $1.3^{58}$ was employed using a simulated annealing algorithm in the torsion angle space with subsequent refinement in explicit water. ${ }^{59}$ The CNS force field used for peptides with explicit hydrogens was supplemented with topologies and parameters developed for an amino acid containing the adamantyl group in $\mathrm{D}$ and L configurations, the linker residue, and D-iGln. An ensemble of structures was generated using an acceptance test, selecting structures with no bond length, valence angle, and NOE violations. The obtained structures were aligned along $\mathrm{C} \alpha$ atoms using Discovery Studio Visualizer 3.5. ${ }^{60}$ The structures were visualized in PyMOL. ${ }^{61}$

Saturation Transfer Difference Experiments. STD spectra were recorded using a pulse sequence with interleaved on- and off-resonance experiments. ${ }^{40}$ Saturation of the lipid resonances was performed using a train of selective Gaussian pulses of $49 \mathrm{~ms}$ duration and $75 \mathrm{~Hz}$ field strength each, separated by a $1 \mathrm{~ms}$ delay. The on-resonance frequency used for saturation of the lipid signals was set to $0.74 \mathrm{ppm}$ for the lipid tail (the fatty acid chain terminal methyl group of DMPC, $14-\mathrm{Me})$ and $5.22 \mathrm{ppm}$ for lipid head groups $\left(2^{\prime} \mathrm{CH}\right.$ of glycerol in DMPC/DHPC). The off-resonance irradiation was applied at $-50 \mathrm{ppm}$ outside the area of NMR resonances. The overall saturation time was $6 \mathrm{~s}$, and 4096 scans were measured. For the epitope mapping analysis, ${ }^{41}$ STD signals in the amide region were used because the aliphatic region of the spectra was dominated by the lipid signals. Control experiments were run using aqueous samples of the derivatives and resulted in no signals, thus confirming that no glycopeptide resonances were irradiated. The resulting intensities were normalized to reference spectrum intensities with irradiation at $-50 \mathrm{ppm}$, and STD amplification factors were obtained. Furthermore, the STD amplification factors were corrected for differences in $T_{1}$ relaxation times of the individual ligand protons. ${ }^{62,63} \mathrm{We}$ measured STD signal buildups with saturation times of 1, 4, and $8 \mathrm{~s}$ as shown in Figure S5. For the larger mannosylated compounds, the STD amplification factors started to decline at $8 \mathrm{~s}$ of saturation time. However, the STD signal patterns are similar for all three saturation times. For this reason, only the STD effects observed with $4 \mathrm{~s}$ saturation time are quantified and discussed for epitope mapping.

Determination of ${ }^{1} \mathrm{H} \quad T_{1}$ Relaxation Times. ${ }^{1} \mathrm{H} \quad T_{1}$ relaxation times were determined in inversion recovery experiments. The water signals were suppressed using excitation sculpting. ${ }^{64}$ The interscan delay was set to $6 \mathrm{~s}$. The delay during the inversion recovery measurements was varied between $50 \mathrm{~ms}$ and $5 \mathrm{~s}$, randomly sampled over 16 experiments. The $T_{1}$ relaxation time constants were fitted to the obtained data points with the $T_{1} / T_{2}$ analysis function implemented in TopSpin 3.1.

Paramagnetic Relaxation Enhancement (PRE) Experiments. A manganese(II) ion was used as a water-soluble paramagnetic relaxation agent in the form of a $\mathrm{MnCl}_{2}$ solution, which was added to the samples containing the $8 \mathrm{mM}$ PGN derivative and $100 \mathrm{mM} \mathrm{DMPC}$ and $200 \mathrm{mM} \mathrm{DHPC}$ at $0.5,1$, and $2 \mathrm{mM}$ concentrations. The effect of the paramagnetic ions was monitored by determination or estimation of $T_{2}$ relaxation times. At low paramagnetic ion concentrations, the ${ }^{1} \mathrm{H} T_{2}$ relaxation times were determined in a Carr-PurcellMeiboom-Gill (CPMG) experiment using the pulse sequence, removing $J$ modulation. ${ }^{65}$ The water signal was suppressed using excitation sculpting. ${ }^{64}$ The interscan delay was set to $6 \mathrm{~s}$. The delay in the spin echo was $1.5 \mathrm{~ms}$, and the total echo time was varied between 6 and $1248 \mathrm{~ms}$, randomly sampled over 16 experiments. The $T_{2}$ relaxation time constants were fitted to the obtained data points with the $T_{1} / T_{2}$ analysis function implemented in TopSpin 3.1. For estimation of $T_{2}$ relaxation times at higher paramagnetic ion concentrations, line widths of resonances in the one-dimensional (1D) ${ }^{1} \mathrm{H}$ NMR experiment were determined using the deconvolution routine in TopSpin 3.1. The transverse paramagnetic relaxation enhancement (PRE) rate was defined as a slope of the fitted linear function for the first two points of the titration, for which the attenuation was the largest. ${ }^{37}$

C-Type Lectin Binding. We used plant lectin, concanavalin A (Con A), to assess C-type lectin binding capability of the PGN drug delivery complexes. Since calcium ions are required for concanavalin A binding, samples of the PGN derivatives were prepared with $8 \mathrm{mM}$ glycopeptide, $100 \mathrm{mM} \mathrm{DMPC}$, and $200 \mathrm{mM}$ DHPC concentrations in $100 \mathrm{mM} \mathrm{NH}_{4} \mathrm{OAc}$ buffer and $2 \mathrm{mM} \mathrm{CaCl}_{2}$ as a reference without and with the addition of concanavalin A ( $1 \mathrm{mM})$. These NMR experiments were carried out in a $1 \mathrm{~mm}$ capillary NMR probe measured in a 1 $\mathrm{mm}$ triple-inverse room-temperature probe with gradients (Bruker TXI-Z).

\section{RESULTS AND DISCUSSION}

To gain insight into the interactions between the PGN derivatives and the lipid bilayer, we investigated their DMPCDHPC bicelle model formulations. To focus on the interactions between the peptide and the lipid molecules, the concentrations of the long-chain lipid DMPC (100 mM) were applied in ca. 10-fold excess compared to those of the derivatives (ca. $8 \mathrm{mM}$ ). The ${ }^{1} \mathrm{H}$ NMR spectra of the formulations are dominated by the lipid signals in the aliphatic region, and only the amide group signals of the PGN derivatives are present in the low-field ${ }^{1} \mathrm{H}$ NMR region. The signals of the fatty acid chains of DMPC and DHPC are somewhat separated, with the terminal methyl groups fully resolved, but the resonances of the polar head groups are in a complete overlap. Despite using nondeuterated lipids, a number of peptide resonances were completely free from the overlap with the larger lipid signals in the aliphatic region as illustrated in the ${ }^{1} \mathrm{H}$ NMR spectrum of $\mathrm{D}-\mathrm{Ad}_{1} \mathrm{Tp}$ in Figure $\mathrm{S} 1$. These nonoverlapping resonances in the aliphatic region and the amide signals were assigned, and their $T_{1}$ or $T_{2}$ relaxation times needed for the evaluation of STD and PRE data were determined. It was, however, not possible to detect STD effects for resonances in the aliphatic region because STDs have only a fraction of the original signal intensity and drown in the presence of the larger lipid signals. Also, at high paramagnetic ion concentrations, $T_{2}$ relaxation times could only be estimated from line widths in the aliphatic region.

Detection of the Interaction by Monitoring the Sign of the Transient NOE. Since the ${ }^{1} \mathrm{H}$ NMR spectrum did not 
(a)

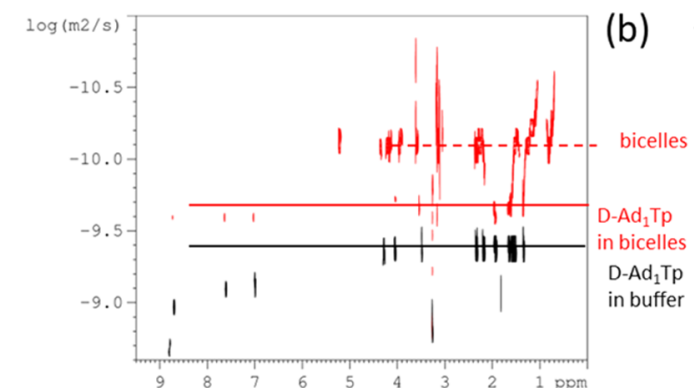

(c)

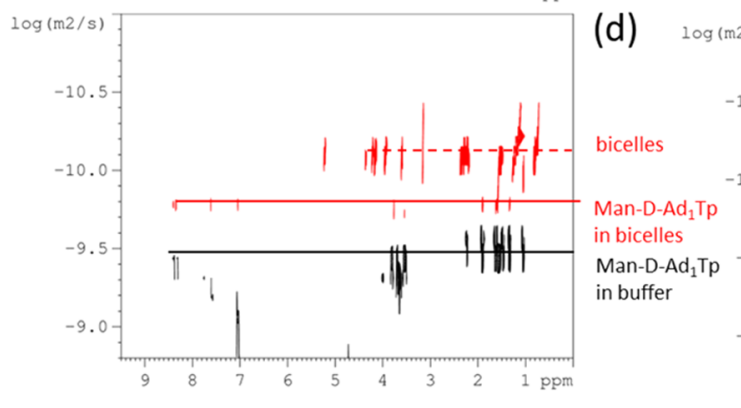

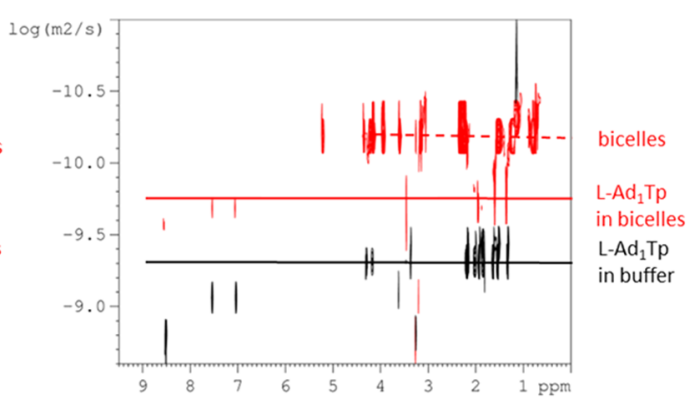
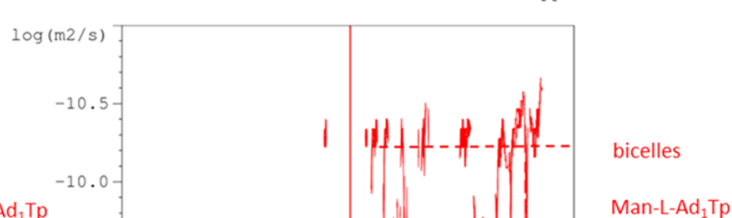

Man-L-Ad ${ }_{1} \mathrm{Tp}$ in bicelles

Man-L-Ad ${ }_{1} \mathrm{Tp}$ in buffer

(e)

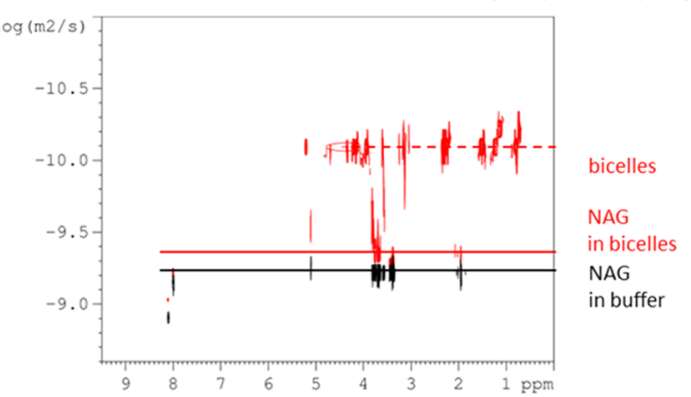

Figure 1. DOSY plot of compounds at $25^{\circ} \mathrm{C}$ : (a) D-Ad $\mathrm{Ad}_{1} \mathrm{Tp},(\mathrm{b}) \mathrm{L}-\mathrm{Ad}_{1} \mathrm{Tp},(\mathrm{c}) \mathrm{Man}-\mathrm{D}-\mathrm{Ad}_{1} \mathrm{Tp},(\mathrm{d}) \mathrm{Man}-\mathrm{L}-\mathrm{Ad}_{1} \mathrm{Tp}$, and (e) NAG in the presence (red) and absence (black) of bicelles.

reveal significant line broadening of the peptide signals (Figure S2), we recorded 2D NOESY spectra to determine the sign of the NOEs, which is dependent on the global motional characteristics of the assembly and thus the size of the molecular species. Positive NOEs were detected in aqueous solutions, and large, negative NOEs were observed in the presence of the bicelles as depicted in Figure S3. Observation of the NOE sign change from positive to negative indicated interaction of the PGN derivatives with the lipid bilayer. As a control, we used $\mathrm{N}$-acetyl-D-glucosamine (NAG), which is an uncharged polar molecule and therefore expected to have little or no interaction with the fatty acid side chains of the lipid molecules. For NAG, weakly positive NOEs were observed both in the aqueous solution and in the presence of bicelles as shown in Figure S2e.

Only a single set of resonances was observed for the PGN derivatives in the presence of bicelles, which indicates that the molecules are in fast exchange between the lipid bilayer and the aqueous solution on the chemical shift time scale. The detected NOEs under fast exchange conditions are transferred NOEs ${ }^{36}$ carrying information on the bound conformation. No intermolecular NOE contacts between the PGN derivatives and the bilayer were detected in the NOESY or the rotating frame NOESY (ROESY) experiment. This is, however, not unusual for molecules with weak to intermediate affinity to membranes. $^{30}$

Thus, the PGN derivatives are partially bound to the bilayer and partially solvated in the aqueous phase in a dynamic equilibrium. In a liposome-based delivery system to be used in a vaccination context, the PGN derivatives would be likely in part intercalated into the lipid bilayer and in part entrapped in the inner aqueous compartment.

Determination of the In Situ Encapsulation Efficiency by the Measurement of Translational Diffusion. Changes in translational diffusion coefficients in the presence of bicelles compared to those in the aqueous solution of the PGN derivatives provided another piece of evidence of the interaction. DOSY plots of the PGN derivatives in bicelles show a significant drop in the translational diffusion coefficient compared to that in the aqueous solution (Figure 1). The diffusion coefficient of NAG in an aqueous buffer solution is very similar to its value in the presence of bicelles (Figure 1e), except for a small difference probably due to different sample viscosity.

A single set of signals was observed in the diffusion dimension for each species; thus, the derivatives are in fast exchange not only on the chemical shift scale but also on the diffusion time scale. This provided an opportunity to use the diffusion coefficients to quantify the lipid encapsulation efficiency.

The decays of the peptide signals generated in the diffusion experiment and used in a numerical fitting are monoexponential when plotted against the square of the gradient strength (Figure S4), which indicates that the exchange regime on the diffusion time scale is fast compared to the inverse of the diffusion delay $(200 \mathrm{~ms})$. Assuming a two-site exchange of the 
derivatives between the bicelle-bound and free solvated forms, we can use the diffusion constants of the different molecular species to estimate the fraction of peptides bound to the lipid bilayer and to calculate the encapsulation efficiency. ${ }^{30,66,67}$ Under fast exchange conditions, the observed diffusion coefficient is a population average of the diffusion coefficients of the molecules freely diffusing in the aqueous solution and that of the molecules bound to the bicelle

$$
D_{\text {obs }}=p_{\text {lipid }} D_{\text {bicelle }}+\left(1-p_{\text {lipid }}\right) D_{\text {free }}
$$

where $D_{\text {obs }}$ is the observed diffusion coefficient of the compound in the presence of bicelles, $D_{\text {bicelle }}$ is the diffusion coefficient of the bicelle as measured on the DMPC terminal methyl signal, $D_{\text {free }}$ is the diffusion coefficient of the compound in the aqueous phase, and $p_{\text {lipid }}$ is the fraction of compounds encapsulated in lipid bicelles.

Furthermore, the measured diffusion coefficients of the PGN derivatives are corrected for the obstruction effect arising from the reduced volume available for the assemblies to diffuse, thus providing the diffusion coefficient at infinite dilution

$$
D_{\text {obs }}=D_{\text {measured }}(1-2 k \Phi)
$$

where $D_{\text {obs }}$ is the diffusion coefficient at infinite dilution; $D_{\text {measured }}$ is the measured diffusion coefficient; $k$ is a shapedependent factor assuming the near-spherical shape of the bicelles; ${ }^{68}$ and $\Phi$ is the volume fraction, which was approximated by the weight fraction of phospholipids excluding the monomer DHPC. ${ }^{30,48}$ Finally, to account for different viscosities in the aqueous and the bicelle solutions, a correction factor was used based on the ratio of the diffusion coefficients of water in the two solutions $\left(234 \mu \mathrm{m}^{2} / \mathrm{s}\right.$ in water and $253 \mu \mathrm{m}^{2} / \mathrm{s}$ in bicelles with $\left.q=0.5^{66}\right)$.

Thus, the fraction of the compounds incorporated in the lipid bicelles $\left(p_{\text {lipid }}\right)$ and their partition coefficient $\left(K_{\mathrm{p}}\right)$ can be determined as follows

$$
\begin{aligned}
& p_{\text {lipid }}=\left(D_{\text {free }}-D_{\text {obs }}\right) /\left(D_{\text {free }}-D_{\text {lipids }}\right) \\
& K_{\mathrm{p}}=p_{\text {lipid }} /\left(1-p_{\text {lipid }}\right)
\end{aligned}
$$

The diffusion properties of the bicelle can be estimated by the diffusion of DMPC, which is only present in the bicelles and does not dissolve in the aqueous solution as opposed to DHPC. ${ }^{66}$ The diffusion constant of the bicelles without added compounds was $53.4 \pm 0.4 \mu \mathrm{m}^{2} / \mathrm{s}$ at room temperature and $59.2 \pm 0.7 \mu \mathrm{m}^{2} / \mathrm{s}$ at $37^{\circ} \mathrm{C}$. These values agree well with $5.2 \times$ $10^{-11}$ and $6.4 \times 10^{-11} \mathrm{~m}^{2} / \mathrm{s}$ found in the literature. ${ }^{69,70}$ The hydrodynamic radii of bicelles with $q=0.5 \mathrm{DMPC} / \mathrm{DHPC}$ ratio has been reported in the range of $3-4.5 \mathrm{~nm}^{49}$ at temperatures between 18 and $40{ }^{\circ} \mathrm{C}$.

Numerical diffusion constants determined by fitting and encapsulation efficiencies at 25 and $37{ }^{\circ} \mathrm{C}$ are shown in Tables 1 and 2, respectively. The bicelle diffusion constants did not change significantly when NAG was added. In the presence of the PGN compounds, the bicelle diffusion constants decreased for the $\mathrm{L}$ diastereoisomers, indicating that the bicelles became larger at room temperature and did not significantly change for the diastereoisomers at room temperature. At $37{ }^{\circ} \mathrm{C}$, the bicelle diffusion constants increased, pointing to the presence of smaller bicelles with a larger increase for Man-D-Ad $1 \mathrm{Tp}$. The increase of bicelle size upon the insertion of peptides has been frequently reported. At $25{ }^{\circ} \mathrm{C}$, the DMPC diffusion increases to $3.5 \times 10^{-11} \mathrm{~m}^{2} / \mathrm{s}$ with C-peptide ${ }^{71}$ and to $2.4 \times 10^{-11} \mathrm{~m}^{2} / \mathrm{s}$

\begin{tabular}{|c|c|c|c|c|c|}
\hline compounds & $\begin{array}{c}D_{\text {bicelle }}\left(10^{-12}\right) \\
\left(\mu \mathrm{m}^{2} / \mathrm{s}\right)\end{array}$ & $\begin{array}{c}D_{\text {free }}\left(10^{-12}\right) \\
\left(\mu \mathrm{m}^{2} / \mathrm{s}\right)\end{array}$ & $\begin{array}{c}D_{\text {obs }}\left(10^{-12}\right) \\
\left(\mu \mathrm{m}^{2} / \mathrm{s}\right)\end{array}$ & $p_{\text {lipid }}$ & $K_{\mathrm{p}}$ \\
\hline $\mathrm{D}-\mathrm{Ad}_{1} \mathrm{Tp}$ & $55.0 \pm 0.2$ & $383 \pm 1.7$ & $215 \pm 7.1$ & 0.32 & 0.47 \\
\hline $\mathrm{L}-\mathrm{Ad}_{1} \mathrm{Tp}$ & $43.5 \pm 0.7$ & $376 \pm 1.7$ & $162 \pm 0.1$ & 0.49 & 0.96 \\
\hline $\begin{array}{l}\text { Man-D- } \\
\text { Ad }_{1} \mathrm{Tp}\end{array}$ & $58.1 \pm 0.2$ & $268 \pm 4.3$ & $151 \pm 4.8$ & 0.19 & 0.23 \\
\hline $\begin{array}{l}\text { Man-L- } \\
\text { Ad }_{1} \mathrm{Tp}\end{array}$ & $41.8 \pm 0.1$ & $283 \pm 7.7$ & $185 \pm 3.5$ & 0.24 & 0.32 \\
\hline NAG & $54.6 \pm 0.2$ & $536 \pm 17.3$ & $388 \pm 2.3$ & 0.01 & 0.01 \\
\hline
\end{tabular}

Table 1. Diffusion Coefficients Determined in the Aqueous and the Lipid Phase, the Derived Lipid-Bound Fraction, and Partition Coefficients at $25{ }^{\circ} \mathrm{C}$

Table 2. Diffusion Coefficients Determined in the Aqueous and the Lipid Phase, the Derived Lipid-Bound Fraction, and Partition Coefficients at $37^{\circ} \mathrm{C}$

\begin{tabular}{lccccc} 
& $\begin{array}{c}D_{\text {bicelle }} \\
\left(10^{-12}\right) \\
\left(\mu \mathrm{m}^{2} / \mathrm{s}\right)\end{array}$ & $\begin{array}{c}D_{\text {free }}\left(10^{-12}\right) \\
\left(\mu \mathrm{m}^{2} / \mathrm{s}\right)\end{array}$ & $\begin{array}{c}D_{\text {obs }}\left(10^{-12}\right) \\
\left(\mu \mathrm{m}^{2} / \mathrm{s}\right)\end{array}$ & $p_{\text {lipid }}$ & $K_{\mathrm{p}}$ \\
compounds & $82.1 \pm 0.3$ & $622 \pm 10.0$ & $304 \pm 8.3$ & 0.51 & 1.05 \\
D-Ad ${ }_{1} \mathrm{Tp}$ & $86.9 \pm 0.6$ & $537 \pm 15.8$ & $252 \pm 11.2$ & 0.56 & 1.26 \\
$\mathrm{~L}_{-} \mathrm{Ad}_{1} \mathrm{Tp}$ & 852 & & \\
$\begin{array}{c}\text { Man-D- } \\
\text { Ad }_{1} \mathrm{Tp}\end{array}$ & $107.5 \pm 1.8$ & $480 \pm 10.6$ & $288 \pm 15.2$ & 0.43 & 0.75 \\
$\begin{array}{c}\text { Man-L- } \\
\text { Ad } \mathrm{Tp}\end{array}$ & $86.6 \pm 0.2$ & $495 \pm 12.1$ & $262 \pm 15.9$ & 0.42 & 0.72 \\
NAG & $55.7 \pm 0.2$ & $549 \pm 9.8$ & $469 \pm 20.0$ & 0.02 & 0.02 \\
\hline
\end{tabular}

with viscosinamide. ${ }^{72}$ At $37^{\circ} \mathrm{C}$, it increases to $2.5 \times 10^{-11} \mathrm{~m}^{2} /$ $\mathrm{s}$ in the presence of penetratin ${ }^{73}$ to $2.8 \times 10^{-11} \mathrm{~m}^{2} / \mathrm{s}$ with motilin ${ }^{66}$ and to $5.0 \times 10^{-11} \mathrm{~m}^{2} / \mathrm{s}$ with KALP21. ${ }^{69}$ These peptides are, however, much larger than the PGN compounds and are known to penetrate the membrane. The bicelle diffusion constant was found to be $6.21 \times 10^{-11} \mathrm{~m}^{2} / \mathrm{s}$ in the presence of amantadine ${ }^{30}$ at $25{ }^{\circ} \mathrm{C}$, however, with $q=0.3$ DMPC/DHPC bicelles.

The lipid incorporation values are between 19 and $49 \%$ at room temperature and 42 and $56 \%$ at $37{ }^{\circ} \mathrm{C}$. In comparison, the incorporation efficiency of the peptidoglycan monomer ${ }^{74}$ fragment isolated from Gram-negative bacteria embedded into lipid bilayers is $16-23 \%$ as determined by an high-performance liquid chromatography (HPLC)-based method. ${ }^{75}$ Thus, a significant increase in the membrane incorporation efficiency could be achieved by the inclusion of the lipophilic adamantane group compared to the unsubstituted peptidoglycan monomer immune modulator, which improves ligand density on the surface of lipid nanoparticle formulations. Lipid entrapments of 22 and $35 \%$ were determined for isomers of the adamantyl compounds with adamantyl-2-yl attachment, D$\mathrm{Ad}_{2} \mathrm{Tp}$ and $\mathrm{L}-\mathrm{Ad}_{2} \mathrm{Tp},{ }^{75}$ respectively (depending on the method of preparation, 23 and $30 \%$ were also measured). The lipid-topeptide ratio in these preparations was closer to $1: 1$ rather than 10:1 in the NMR investigation, which could explain lower incorporation values. Membrane partitioning $\left(K_{\mathrm{p}}\right)$ of spinlabeled amantadine was found to be 11.2 at $45{ }^{\circ} \mathrm{C}$ in DMPC vesicles using electron paramagnetic resonance $(\mathrm{EPR})^{29}$ and 27.6 in DMPC and 37.8 in 1-palmitoyl-2-oleoyl-sn-glycero-3phosphocholine (POPC) lipids using NMR, ${ }^{30}$ which are much larger than the partition coefficients determined here. Amantadine is, however, much smaller in size than the derivatives studied here and has only a single charged group.

By comparing the values, two observations may become relevant for the design of these molecules. Previously, we have shown $^{34}$ that the peptide co-stimulation activity to LPS on 
macrophages was further increased by the incorporation of the compounds into phosphatidylcholine lipid bilayers. Only L$\mathrm{Ad}_{1} \mathrm{Tp}$ and Man-L-Ad $\mathrm{Tp}$ were investigated in the in vitro assay for this effect. Albeit that the adjuvant effect of Man-L$\mathrm{Ad}_{1} \mathrm{Tp}$ both in the aqueous solution and in liposomes was better, the relative increase of the adjuvant effect upon the incorporation of $\mathrm{L}-\mathrm{Ad}_{1} \mathrm{Tp}$ compared to that in the aqueous solution was more significant. Thus, $\mathrm{L}-\mathrm{Ad}_{1} \mathrm{Tp}$ profited more from the lipid delivery system than Man-L-Ad ${ }_{1} \mathrm{Tp}$ and this may be related to the higher lipid incorporation efficiency of the nonmannosylated compound compared to that of the derivative with the mannose group.

We may also see a slight influence of the chirality of the adamantyl group attachment. Immune co-stimulation activities to LPS in vitro were also shown to be dependent on the chirality of the adamantyl group attachment, ${ }^{34}$ but this is likely related to molecular details of the receptor interaction. Of the nonmannosylated diastereoisomer pair, $\mathrm{D}-\mathrm{Ad}_{1} \mathrm{Tp}$ appears to interact weaker with the lipid bilayer than $\mathrm{L}-\mathrm{Ad}_{1} \mathrm{Tp}$. This difference is slightly more pronounced at low temperature (32 vs $49 \%$ ) and present to much less degree at high temperature (51 vs 56\%). For the mannosylated derivatives, the encapsulation between the diastereoisomers differs less at room temperature (19 vs $24 \%$ ) and is similar at $37{ }^{\circ} \mathrm{C} \mathrm{(43} \mathrm{vs}$ $42 \%)$. Nevertheless, the differences might be attributable to the experimental uncertainty of the lipid incorporation efficiencies.

Characterization of the Membrane-Associated Conformation with Transferred NOEs. To get insight into the range of conformations available for the PGN derivatives in the lipid bilayer bound state, we used transferred NOEs detected in the presence of bicelles at room temperature (Table S1) to calculate the structures associated with the membrane. For D$\mathrm{Ad}_{1} \mathrm{Tp}$ and $\mathrm{L}-\mathrm{Ad}_{1} \mathrm{Tp}, 25$ and $14{ }^{1} \mathrm{H}-{ }^{1} \mathrm{H}$ distances were extracted, respectively, while for Man-D-Ad ${ }_{1} \mathrm{Tp}$ and for Man-L$\mathrm{Ad}_{1} \mathrm{Tp}$, the distances were 26 in both cases. The calculated ensembles are shown in Figure 2.

The distance restraints derived from transferred NOEs do not determine a single conformation but rather a range of structures. However, certain trends can be observed. For the nonmannosylated derivatives, the backbone was more or less extended for the majority of $\mathrm{L}-\mathrm{Ad}_{1} \mathrm{Tp}$ structures (Figure $2 \mathrm{a}$ ), while, for $\mathrm{D}-\mathrm{Ad}_{1} \mathrm{Tp}$, the C-terminus tends to bend back to the $\mathrm{N}$-terminus (Figure $2 \mathrm{~b}$ ). This bent conformation in $\mathrm{D}-\mathrm{Ad}_{1} \mathrm{Tp}$ is enforced by a series of unique restraints from the Ala-NH to the Ada side chain (denoted in bold in Table S1). While the structures of the nonmannosylated derivatives depicted in Scheme 1 show neutral forms, the peptide termini are likely zwitterionic in the aqueous solution; thus, this bent conformation is likely driven by an intramolecular electrostatic contact between the $\mathrm{N}$-terminal amine group and the $\mathrm{C}$ terminal carboxyl group of the derivative.

In the case of the mannosylated derivatives, the transferred NOEs define extended conformations for Man-D-Ad $\mathrm{T} p$, while Man-L-Ad $1 \mathrm{Tp}$ displayed structures with a somewhat bent backbone, with two of the conformations showing a close contact between their termini as shown in Figure 2c,d. As before, we note that the structures of the mannosylated derivatives in Scheme 1 are depicted in neutral forms, while, in the aqueous solution, ionized states are likely present with a negative charge in the carboxyl group at the C-terminal and the $\mathrm{N}$ termini of the parent compounds are capped with a neutral, albeit polar mannose group. In the strongly bent subpopulation of Man-L-Ad $\mathrm{Ap}_{1}$, the hydroxyl groups of the mannose and the

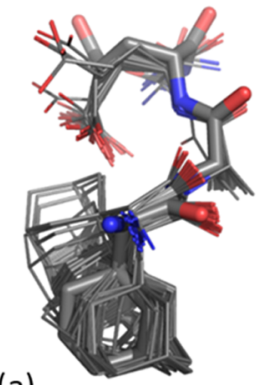

(a)

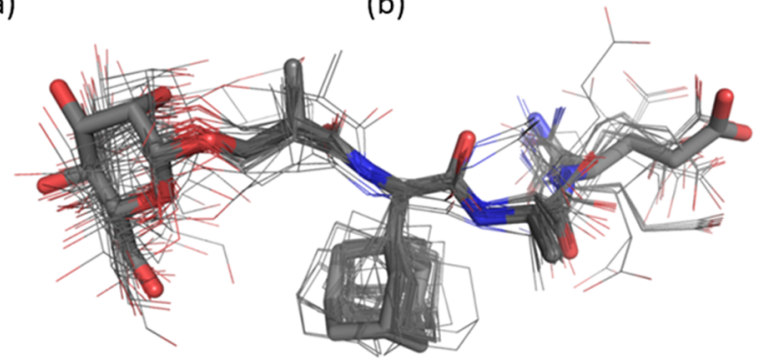

(c)

(d)

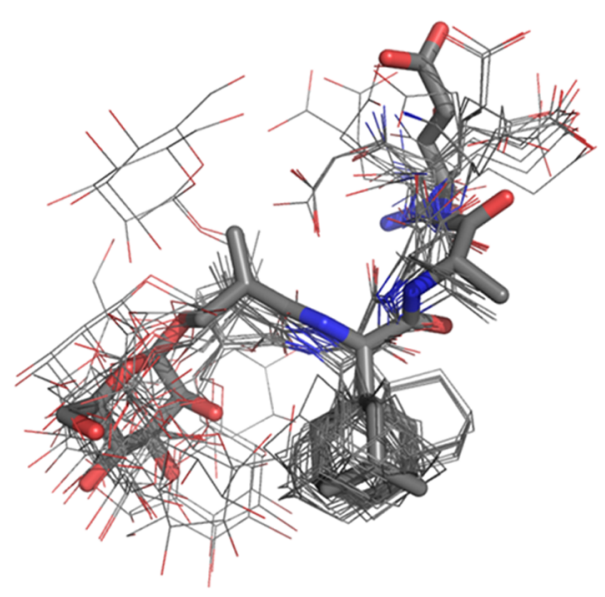

Figure 2. Ensemble of conformations calculated using transferred NOEs for (a) D-Ad $1 \mathrm{Tp}$, (b) L-Ad $1 \mathrm{Tp}$, (c) Man-D-Ad 1 Tp, and (d) Man-L-Ad ${ }_{1} \mathrm{Tp}$. The average structure is shown in sticks.

D-iGln carboxyl group are close enough for a hydrogenbonding interaction, which is absent for Man-D-Ad $\mathrm{T}$ p. For Man-L-Ad $\mathrm{Tp}$, the restraints that are responsible for the bent conformations primarily involve NOEs from the Ala-NH group and the D-iGln side chain to the methyl group of the Lnk residue (denoted in bold in Table S1).

Localization and Mapping of the Orientation within the Bilayer via STD and PREs. While both STDs and PREs can be analyzed to yield quantitative structural, ${ }^{41}$ thermodynamic, ${ }^{76}$ or kinetic $^{77}$ properties, we performed a simpler qualitative evaluation, aiming at a complementary epitope mapping of the lipid-associated and the solvent-accessible parts of the investigated molecules.

STD Spectroscopy. We performed STD experiments to characterize the orientation of the derivatives in the bilayer. We observed that irradiation at the lipid tail (terminal methyl group) and close to the lipid head (glycerol $\mathrm{CH}$ ) groups resulted in different STD amplification patterns. Similar effects have been reported for amantadine in isotropic bicelles, ${ }^{30}$ whereby different STD amplification factors were observed, depending on the lipid groups chosen to be saturated. This effect is likely due to the fact that the spread of saturation is not isotropic throughout the bicelle as a result of inefficient 
(a)

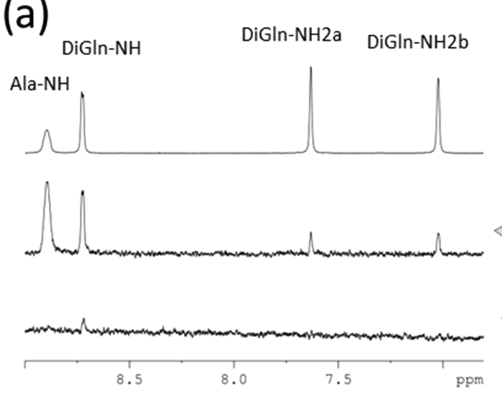

(c)

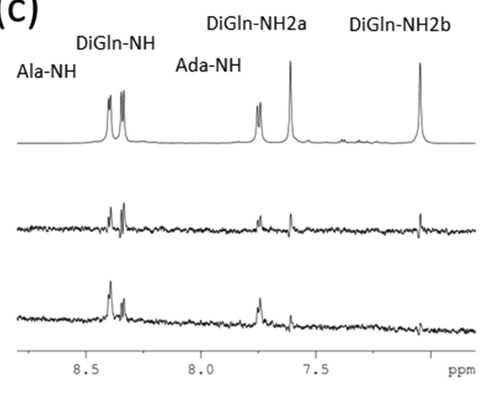

(b)

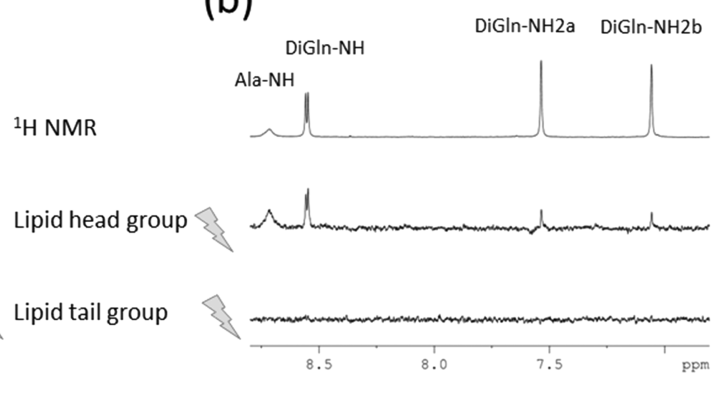

(d) Ala-NH DiGln-NH DiGln-NH2a DiGln-NH2b

${ }^{1} \mathrm{H}$ NMR

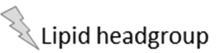

Lipid tail group

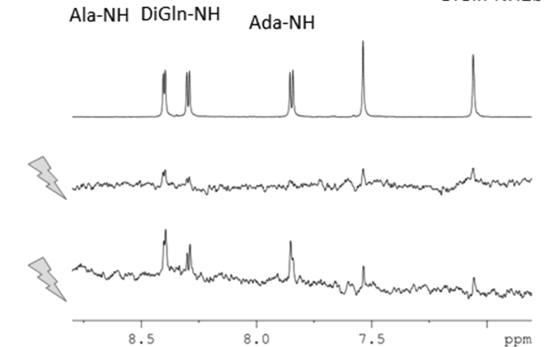

Figure 3. STD with selective irradiation of the resonances of the lipid molecules for compounds (a) D-Ad $T p,(b)$ L-Ad 1 Tp, (c) Man-D-Ad $T$ p, and (d) Man-L-Ad $\mathrm{Tp}$ : the upper trace shows the reference ${ }^{1} \mathrm{H}$ NMR spectrum of the amide signals, the middle trace shows selective irradiation close to the lipid head group (DMPC/DHPC glycerol 2'-CH), and the lower trace shows selective irradiation of the lipid tail (DMPC 14-Me).

spin diffusion. This phenomenon allowed determination of not only the interacting surface of the derivatives but also the part of the lipids where the saturation is transferred from. Hence, the STD experiment in effect was used for mapping the position of the molecules within the lipid bilayer similarly to the case of amantadine. ${ }^{30}$

We performed two types of STD experiments for each derivative as shown in Figure 3: in one experiment, the terminal DMPC methyl signal was saturated, and in the second experiment, the resonance of the $2^{\prime} \mathrm{CH}$ group of the glycerol backbone representing the overlapping signals of DMPC and DHPC molecules close to the lipid head was irradiated. Identical reference experiments were performed using the aqueous samples of the PGN derivatives, which produced no signal (data not shown). Other lipid resonances were in an overlap with the peptide signals that was too strong to allow selective saturation. Only the amide group STDs of the peptides were evaluated as the weak STD signals in the aliphatic region were drowned by the intense signals from the lipid molecules, preventing their detection. Special care needs to be taken to address artifacts distorting STD effects arising from differential relaxation times. ${ }^{62}$ The $T_{1}$ relaxation times for the corresponding amide resonances were suggested as a correction factor for the final STD amplification factors. ${ }^{63}$ However, as the $T_{1}$ relaxation times (Table S2) were very similar to each other for a given derivative, they did not affect significantly the STD amplification factors (Table S3).

Looking at the STD spectra, we can make one important qualitative observation at this stage. For the nonmannosylated tripeptides, no or very little STD signal was obtained when the lipid tail was irradiated as compared to experiments with irradiation close to the lipid head groups (except for a small signal at $\mathrm{D}-\mathrm{iGln}-\mathrm{NH}$ in $\mathrm{D}-\mathrm{Ad}_{1} \mathrm{Tp}$ ). This indicates that the nonmannosylated compounds are not in contact with the inner parts of the bilayer. For the mannosylated compounds, STD signals were detected in both experiments, which implies that the mannosylated derivatives are more immersed into the bilayer than their parent compounds.

Mapping of the STD amplification factors on the structures as shown in Figure 4 allows the qualitative characterization of the location and orientation of the derivatives within the lipid bilayer. Two major conclusions can be drawn. First, the STD signal intensity observed for the side-chain $\mathrm{D}-\mathrm{iGln}-\mathrm{NH}_{2}$ groups is less intense than that of the STD for the other groups. Also,

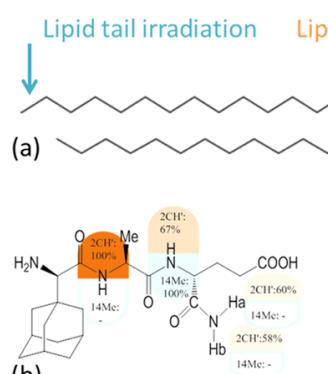

(b)

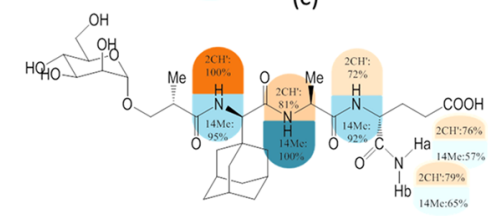

(e)

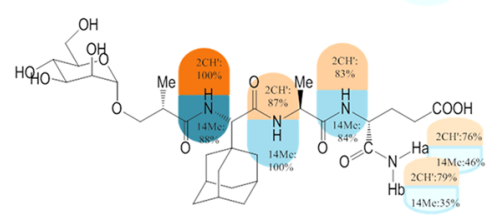

Figure 4. STD signal intensities mapped down onto the structure for compounds (a) D-Ad $\mathrm{Tp}$, (b) L-Ad 1 Tp, (c) Man-D-Ad $\mathrm{Tp}$, and (d) Man-L-Ad 1 p. The upper value indicates the STD intensity with irradiation close to the lipid head group, while the lower value shows the STD signal with lipid tail saturation. 


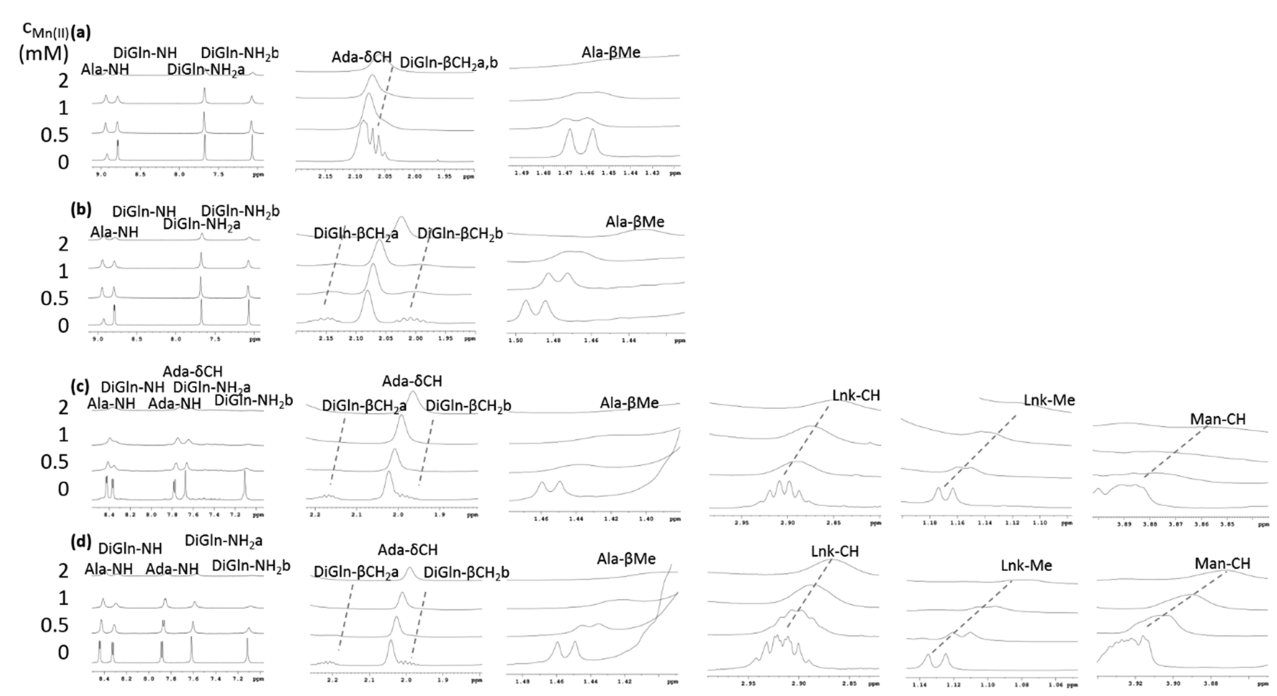

Figure 5. Broadening of ${ }^{1} \mathrm{H}$ NMR resonances during the titration with paramagnetic manganese(II) ions for (a) D-Ad $1 \mathrm{Tp},(\mathrm{b}) \mathrm{L}-\mathrm{Ad} \mathrm{d}_{1} \mathrm{Tp},(\mathrm{c}) \mathrm{Man}-\mathrm{D}-$ $\mathrm{Ad}_{1} \mathrm{Tp}$, and (d) Man-L-Ad $\mathrm{Tp}$.

it is less intense with lipid tail irradiation than with saturation close to the lipid head group. This indicates that the D-iGln residue does not appear to be in close contact with the bilayer. Second, when resonances close to the lipid head groups are saturated, the most intense STD signal is detected at the Ala$\mathrm{NH}$ group for the nonmannosylated compounds, while the Ada- $\mathrm{NH}$ moiety received most saturation for the derivatives with mannose groups. Since the lipid resonances dominate the aliphatic region, the mannose and adamantyl groups are in effect invisible with STD mapping. The Ala-NH and Ada-NH groups are the closest to the adamantyl moiety with observable STDs to serve as reporter groups. These groups displaying the strongest STDs thus indicate that the adamantyl group is inserted into the bilayer deepest, at least at the level of the glycerol moiety.

To conclude the findings of the STD studies, the nonmannosylated parent compounds interact only with the upper layer of the lipid bilayer, while the derivatives with mannose groups appear to be more immersed into the membrane. In all cases, the PGN fragment cargo is closest to the surface according to small STDs on $\mathrm{D}-\mathrm{iGln}-\mathrm{NH}_{2}$ groups; thus, the cargo PGN peptide fragment is available for interactions with innate immune receptors in the membrane surface, e.g., for activation of the NOD2 receptor, which recognizes the PGN fragment ${ }^{9}$ and induces a Th1-type immune response.

Water-Soluble Paramagnetic Probes. To investigate the parts of the PGN derivatives that are not visible using STD due to the dominance of strong lipid resonances in the aliphatic region, that is, the adamantyl group and the mannosyl group, we used PRE induced by water-soluble paramagnetic probes, specifically by manganese(II) ions. Manganese(II) ions ${ }^{44}$ induce strong PREs due to five unpaired electrons and long electron relaxation times, but they have an isotropic electron $g$ tensor and thus do not generate pseudocontact shifts. We monitored PRE-induced bleaching effects during titration with paramagnetic ions for various reporter groups in the derivatives as shown in Figure 5.

We also quantified the bleaching effect defined as the attenuation of $T_{2}$ relaxation times (Figure 6), which were either measured in CPMG experiments or estimated from line widths during the titration (Table S4). In the case of many

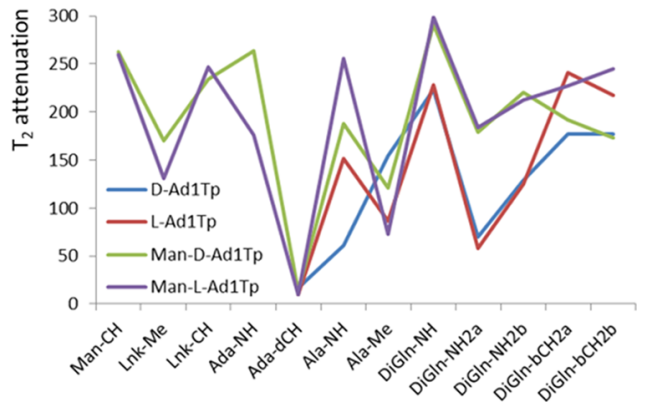

Figure 6. Attenuation of the relaxation times upon the addition of paramagnetic ions as measured by the slope of the linear function fitted for $T_{2}$ relaxation times at the first two points of the titration, that is, at 0 and $0.5 \mathrm{mM}$ of paramagnetic ion concentrations.

resonances, the $T_{2}$ relaxation times did not decrease linearly with increasing paramagnetic ion concentrations. The change between the first two points of titration (for which no paramagnetic ion was present, followed by the addition of 0.5 mM manganese(II) ions) was the largest, while, for the remaining titration points, diminishing reduction of $T_{2}$ relaxation times was observed. For this reason, the slope of the linear function fitted for the first two points was used to define the attenuation of the $T_{2}$ relaxation times.

We found that the resonances of $\mathrm{D}-\mathrm{iGln}-\beta \mathrm{CH}_{2}$ and the mannose group (Figure 5, columns 2 and 6, respectively) quickly decline (large $T_{2}$ attenuation, Figure 6), which indicates that these terminal groups are solvent-exposed. Such localization of D-iGln on the surface is also in agreement with the result of the STD epitope mapping. In contrast, the signals of the adamantyl moiety, Ada- $\delta \mathrm{CH}$, are hardly affected by the addition of the paramagnetic ions and display similar line widths and intensities at high or low paramagnetic ion concentrations (Figure 5, column 2). This indicates that the adamantyl group is immersed in the lipid bilayer, which protects it from the relaxation effects of the water-solvated paramagnetic ions (low $T_{2}$ attenuation, Figure 6). Between the two extremes, the attenuation is gradually changing: the resonances of the Ala- $\beta \mathrm{Me}$ group (Figure 5, column 3 ) and the linker residue (Lnk) (Figure 5, column 4) show intermediate bleaching effects (Figure 6). Comparison of the bleaching 
effects of the amide resonances in Figure 6 shows that for all derivatives the $\mathrm{D}$-iGln- $\mathrm{NH}$ shows the fastest initial decrease in $T_{2}$ relaxation times (large $T_{2}$ attenuation), resulting in broadening of line width and the loss of intensity upon increasing paramagnetic ion concentrations (Figure 5, column 1). This further confirms the solvent exposure of the PGN fragment cargo.

The PRE data indicates that the targeting mannosyl group and the PGN fragment cargo of the derivatives are available for interaction with cell surface receptors. These findings are in line with previous observations for related PGN-based adjuvants with adamantyl-2-yl attachment, ${ }^{78}$ whereby the mannose group was found on the surface of liposomes by dynamic light scattering and atomic force microscopy. Specific binding of the mannosyl group on cell surface receptors ${ }^{79}$ such as the mannose receptor (MR) could explain improved immune stimulatory properties of the mannosylated derivatives. The $\mathrm{MR}^{80}$ is a C-type lectin receptor expressed in macrophages, which facilitates the internalization of microorganisms and glycoproteins with terminal mannose residues. Recognition by the MR could increase the uptake of the mannosylated derivatives and hence contribute to their immune stimulation properties.

We found that the PRE data indicated that the adamantyl group is intercalated into the lipid bilayer and serves as an anchor to the membrane. In comparison, amantadine was located in the head group region of phosphocholine membranes at the surface of the lipid bilayer, as observed by neutron and X-ray diffraction. ${ }^{28}$ However, occasionally, it was also shown to penetrate the deeper regions of the bilayer, which was also confirmed by EPR. ${ }^{29}$ In a liquid-state NMR study, ${ }^{30}$ it was possible to perform irradiation of a number of lipid resonances, enabling the more precise mapping of amantadine by STD. This showed that the drug is distributed in a wide region, starting from the glycerol $\mathrm{CH}_{2}$ group near the negatively charged phosphate group and the entire hydrocarbon region. Thus, it appears that the adamantane group can position itself in various regions of the membrane. The surface location of amantadine is probably driven by electrostatic attraction between the negatively charged phosphate head groups of the lipids and the positive charge of the amine group on amantadine, similarly to the case of the nonmannosylated PGN derivatives, which also have a positively charged terminal amine group. Deeper penetration of amantadine into the aliphatic lipid chain region is likely driven by the strong hydrophobicity of the adamantane group. This interaction might also become more dominant for the mannosylated derivatives, in which the charged amine groups at the $\mathrm{N}$ termini are capped by the neutral mannose groups.

As we noted earlier, the nonmannosylated compounds are incorporated more efficiently into the bilayer at both temperatures, while attachment of the mannose group reduces the incorporation efficiency. Taking into consideration the results obtained regarding the conformation, localization, and orientation of the derivatives in the bilayer, we propose the following molecular interpretation for this finding. The nonmannosylated derivatives are in zwitterionic forms, containing an ionized $\mathrm{N}$-terminal amine and a C-terminal carboxyl group. The interaction of these compounds could involve electrostatic contacts from their charged termini to the positively charged choline groups and the negatively charged phosphate groups in the lipid molecules. In contrast, the mannosylated derivatives bear only a negatively charged C- terminus as the $\mathrm{N}$-terminus is capped by the neutral, albeit polar mannose moiety, making only one ionic interaction possible with the lipids. The presence of two charged groups in the nonmannosylated compounds likely prevents deeper incorporation into the lipid bilayer, explaining the observed STD patterns. However, these two electrostatic interactions in the upper layer of the assembly can reasonably be assumed to result in a stronger interaction with the lipid molecules relative to the mannosylated compounds, where only one strong electrostatic interaction is present, thus leading to stronger incorporation efficiencies of the nonmannosylated compounds.

Furthermore, we observed that the chirality of the adamantyl group influences the incorporation efficiency, which might be correlated with the membrane-associated conformation in the following way. D-Ad $\mathrm{Tp}$ interacts weaker with the lipid bilayer than $\mathrm{L}-\mathrm{Ad}_{1} \mathrm{Tp}$, with a most pronounced difference at low temperature (32 vs $49 \%$ ) and a smaller distinction at high temperature (51 vs $56 \%$ ). At room temperature, D- $\mathrm{Ad}_{1} \mathrm{Tp}$ adopts a bent conformation, which may be attributed to an ionic interaction between the charged $\mathrm{C}$ - and $\mathrm{N}$-terminal groups. Such an intramolecular contact might prevent or at least compete with the charged terminals engaging in intermolecular interactions with the lipid molecules, and this could explain the weaker encapsulation into the bilayer. For the mannosylated derivatives, the encapsulation between the diastereoisomers differs less at room temperature (19 vs $24 \%$ ) and is similar at $37^{\circ} \mathrm{C}$ (43 vs $42 \%$ ). A subpopulation of Man-L$\mathrm{Ad}_{1} \mathrm{Tp}$ determined at room temperature also displayed a bent backbone enabled by an intramolecular hydrogen bond between the termini. Similar to the reasoning above, these groups may not be fully available for intermolecular electrostatic interactions with polar lipid groups, which might explain lower encapsulation. These observations and explanations are in agreement with reports showing that the lipophilicity of drug molecules has been found to be affected by the formation of intramolecular hydrogen bonds, which shields the hydrogenbond donor and acceptor groups from intermolecular interactions. ${ }^{81}$ In the case of the PGN molecules, the interaction with the lipids is likely driven by a combination of electrostatic interactions, transient hydrogen bonds, and van der Waals forces. Bent intramolecular conformations could affect the availability of the polar groups for the first two, for electrostatic interactions and transient hydrogen bonds, thus leading to the modulation of the incorporation strength of the diastereoisomers.

Binding to CLRs. The adjuvant activity of the adamantylated PGN derivatives likely involves interaction with multiple receptors of the innate immune system; however, these receptors have not yet been unequivocally clarified. Targeting via the mannosyl group requires recognition by cell surface CLRs, such as the MR, that facilitate internalization of microbes by binding of terminal mannose groups and could therefore contribute to the increased uptake and to the augmentation of the adjuvant effect. Targeting was evidenced ${ }^{16}$ by the more effective adjuvant activity of the derivatives with the mannose-targeting group than that of the nonmannosylated parent compounds.

The interaction of CLRs with the mannosyl-targeting group can be mimicked by plant lectins such as concanavalin A (Con A). The specificity of carbohydrate-lectin interactions is often studied using Con A, with detection of the changes in hydrodynamic radius by dynamic light scattering. ${ }^{82}$ Here, we used NMR diffusion experiments to detect changes in 

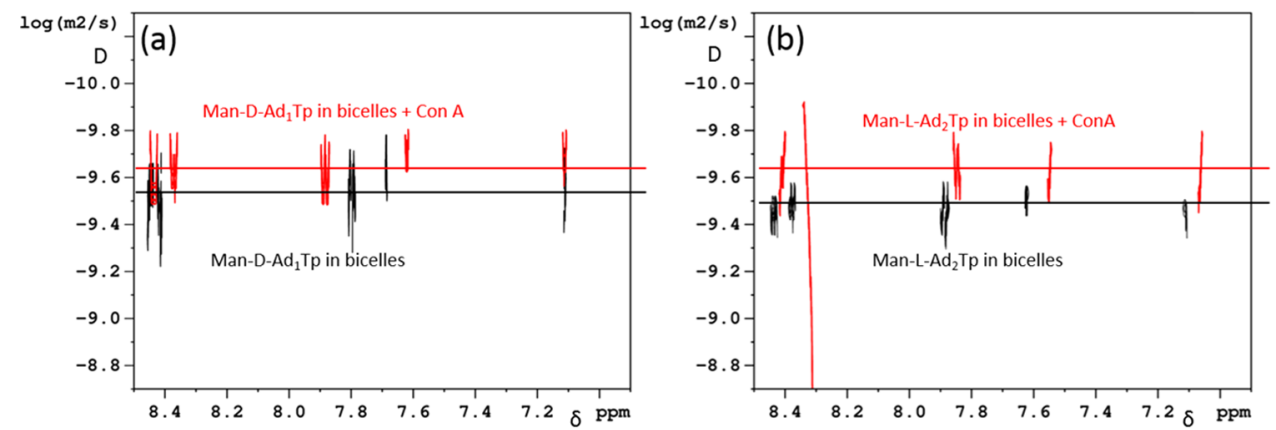

Figure 7. Comparison of DOSY plots for (a) Man-D-Ad $1 \mathrm{Tp}$ and (b) Man-L-Ad $1 \mathrm{Tp}$ in the presence of bicelles (black) and after the addition of 1 $\mathrm{mM}$ Con A (red).

translational diffusion upon the addition of Con A. To see whether the mannosyl group is available for binding to endogenous cell surface receptors, we added $1 \mathrm{mM}$ Con A to the bicelle solutions of the PGN derivatives in the presence of calcium ions and monitored the translational diffusion of the peptides. In both cases, a decrease in the diffusion constants was observed (Figure 7), which was also accompanied by chemical shift changes.

Due to fast exchange between the various glycopeptidecontaining species, only a single apparent diffusion coefficient is observable in the DOSY spectrum, which is a populationaveraged value of the diffusion coefficients of the individual complexes present. The observed decrease in the translational diffusion coefficient of the PGN derivatives may be attributed to the glycopeptide cross-linking bicelles around the lectin, which is able to bind up to four mannosyl groups. This might indicate that the mannosyl moiety of both derivatives incorporated into bilayers is available for binding to cell surface CLRs.

\section{CONCLUSIONS}

PGN fragments such as muramyl peptides ${ }^{83}$ activate the immune system through induction of the NLR pathway, i.e., molecular recognition by intracellular NOD-like receptors. Their activity can be enhanced by other types of PRR, such as toll-like receptor (TLR) activation. Lipids act as TLR ligands and can therefore synergistically enhance the immune response to PGN compounds, which is known as PRR crosstalk. Mannose receptors on the other hand are CLR receptors, which can improve the uptake of the compounds and thereby modulate the immune stimulation. Better incorporation of compounds into the bicelles provides increased ligand density, which positively influences the stimulation of the immune response. The activity is thus controlled by the incorporated PGN-based peptide and the synergistic effect of lipid nanoparticles with an additional effect of the targeting group in the mannosylated compounds.

Lipid incorporation efficiencies of the adamantylated PGN derivatives were between 19 and $49 \%$ and 42 and $56 \%$ at room temperature and $37^{\circ} \mathrm{C}$, respectively, which are much improved compared to the lipid incorporation found for the whole monomeric PGN fragment. The parent adamantylated compounds showed higher lipid encapsulation than their mannosylated conjugates. Immune co-stimulation activities of the derivatives in vitro were amplified upon incorporation into liposomes and more so for the nonmannosylated derivatives than for the compounds with mannose groups, which may be attributed to their higher increase in lipid incorporation efficiency.

It was also found that the nonmannosylated compounds are only in contact with the upper parts of the bilayer, while the derivatives with mannose groups penetrate deeper in the bilayer. In all cases, the PGN cargo fragments and the mannosyl groups of the glycopeptide derivatives are close to the surface of the bilayer. These observations can be rationalized by considering possible electrostatic interactions between the charged groups of the derivatives and those of the lipid molecules. The membrane interaction appears to be slightly influenced by the chirality of the adamantyl group and correlated with the membrane-associated conformations. This effect could be related to the availability of the charged or polar groups for intermolecular interactions with the lipid molecules and thereby explains differences in incorporation efficiency.

On the basis of the findings by NMR, we propose that the mannosylated adamantylated PGN derivatives in liposome formulations could intercalate themselves into the lipid bilayer and would also be present in the inner aqueous compartment due to their solubility in water. In the bilayer bound arrangement, which is depicted in Scheme 2, the targeting

Scheme 2. Proposed Interactions of the Mannosylated PGN Derivatives Embedded in Liposomes with Cell Surface Receptors of Antigen-Presenting Cells (APCs) ${ }^{a}$

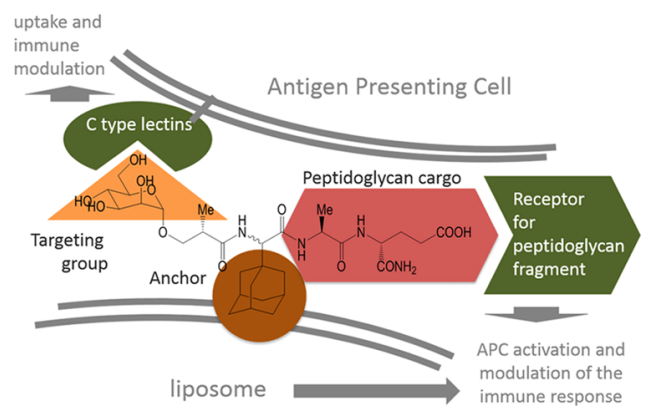

${ }^{a}$ The figure is for the illustration of the mutual arrangement of the components and is not to scale.

mannosyl group is able to interact with cell surface CLRs, while the PGN fragment cargo is available for contact with the cell surface or, after internalization, cytosolic receptors. Thus, we show that different functional groups of the PGN derivatives designed for liposomal delivery are located on the surface of the lipid assembly in an arrangement that enables interactions with the targeted receptors. The conformations of the molecules solvated in the inner aqueous compartment 
might be different than those of the membrane-associated molecules.

By elucidating the molecular details of membrane interactions of adamantylated PGN derivatives, we have taken the first steps toward understanding their lipid formulations, which will enable further modifications to improve the efficiency of membrane encapsulation and the adjuvant activity of this type of molecules. To deliver a cargo in a vaccination context, multiple complementary functionalizations of liposomes are proposed such as sufficient membrane incorporation, active targeting of the cell surface and innate immune signaling receptors, stealth, and controlled release. We conclude that good compounds for functionalizing liposomes should contain lipophilic groups, which could penetrate the lipid bilayer, and homing groups to interact with target receptors and to accumulate the cargo at the site of biological action. The lipophilic adamantane group attached to the peptide backbone in the studied PGN derivatives achieves encapsulation of the compounds into the lipid bilayer, thus providing safe delivery of the payload. Additionally, the attachment of mannose as a ligand for specific receptors expressed on cell surfaces enables active targeting. We confirmed that both the mannose group and the PGN payload are available on the surface of the bilayer. Having achieved sufficient membrane incorporation as well as active targeting for both the uptake and immune response, further improvements could include functionalization to achieve stealth from immune surveillance, e.g., by PEGylation. Furthermore, while the dynamic equilibrium present between the free state in the aqueous solution and the lipid-membrane-bound state of the adamantylated PGM derivatives enables a prolonged release of the payload, this could be improved by incorporating a controlled release mechanism to achieve a burst release triggered at the target site of action.

\section{ASSOCIATED CONTENT}

\section{S1 Supporting Information}

The Supporting Information is available free of charge at https://pubs.acs.org/doi/10.1021/acs.jpcb.0c00029.

${ }^{1} \mathrm{H}$ NMR spectra of $\mathrm{D}-\mathrm{Ad}_{1} \mathrm{Tp}$ complete with the assignment at $37{ }^{\circ} \mathrm{C}$ and $700 \mathrm{MHz}$ (Figure S1); line widths of resonances in ${ }^{1} \mathrm{H}$ NMR spectra (Figure S2); NOESY spectra of the PGN derivatives in bicelles (Figure S3); Stejskal-Tanner plots of PGSTE signal decays (Figure S4); summary of the signal intensities of the transferred NOEs for the PGN derivatives (Table S1); $T_{1}$ relaxation times of the amide resonances (Table S2); STD signal buildup (Figure S5); STD amplification factors corrected with $T_{1}$ relaxation times (Table S3); $T_{2}$ relaxation times for the amide resonances during the $\mathrm{Mn}(\mathrm{II})$ ion titration (Table S4) (PDF)

\section{AUTHOR INFORMATION}

\section{Corresponding Author}

Krisztina Fehér - Department of Organic and Macromolecular Chemistry, Ghent University, 9000 Ghent, Belgium; Heidelberg Institute for Theoretical Studies, 69118 Heidelberg, Germany; Molecular Recognition and Interaction Research Group, Hungarian Academy of Sciences, H-4032 Debrecen, Hungary; (1) orcid.org/0000-0003-0951-2507; Phone: +36-52-512900; Email: feher.krisztina@science.unideb.hu, krisztina.feher@hits.org

\section{Authors}

Rosana Ribić - University Center Varaždin, University North, HR-42 000 Varaždin, Croatia; Heidelberg Institute for Theoretical Studies, 69118 Heidelberg, Germany

Mateja Manček-Keber - Department of Synthetic Biology and Immunology, National Institute of Chemistry, SI-1001 Ljubljana, Slovenia; Heidelberg Institute for Theoretical Studies, 69118 Heidelberg, Germany

Fernando Chain - Department of Organic and Macromolecular Chemistry, Ghent University, 9000 Ghent, Belgium

Davy Sinnaeve - Department of Organic and Macromolecular Chemistry, Ghent University, 9000 Ghent, Belgium; Univ. Lille, Inserm, Institut Pasteur de Lille, CHU Lille, U1167 - Labex DISTALZ - RID-AGE - Risk Factors and Molecular Determinants of Aging-Related Diseases, F-59000 Lille, France; CNRS, ERL9002 - Integrative Structural Biology, F-59000 Lille, France

José C. Martins - Department of Organic and Macromolecular Chemistry, Ghent University, 9000 Ghent, Belgium

Roman Jerala - Department of Synthetic Biology and Immunology, National Institute of Chemistry, SI-1001 Ljubljana, Slovenia

Srdanka Tomic - Department of Chemistry, Faculty of Science, University of Zagreb, HR-10 000 Zagreb, Croatia

Complete contact information is available at:

https://pubs.acs.org/10.1021/acs.jpcb.0c00029

\section{Author Contributions}

R.R. and M.M.-K. contributed equally to this work. The manuscript was written through contributions of all authors. All authors have given approval to the final version of the manuscript.

\section{Notes}

The authors declare no competing financial interest.

\section{ACKNOWLEDGMENTS}

R.R. and S.T. wish to thank the Croatian Science Foundation for support (project IP-2014-09-7899). M.M.-K. and R.J. acknowledge support from the Slovenian Research Agency (program no. P4-0176). F.C. is thankful for the Erasmus Mundus Peace fellowship. D.S. thanks the Research Foundation-Flanders (FWO-Vlaanderen) for a postdoctoral research fellowship and a research grant (1.5.133.13N). K.F. acknowledges the support of the Marie Curie Career Integration Grant (303917 PGN-INNATE) and the Research Grant from the Research Foundation-Flanders (1508414N and $1525517 \mathrm{~N})$, János Bolyai Research Scholarship of the Hungarian Academy of Sciences (BO/004333/18/7) and the New National Excellence Program of Debrecen University (ÚNKP-19-4 Bolyai+). This research was supported by the National Research, Development and Innovation Office of Hungary (grant NKFI/OTKA NN 128368). The authors thank Dr. Ruža Frkanec, Prof. László Szilágyi, and Dr. Niels Geudens for stimulating discussions. The $700 \mathrm{MHz}$ equipment in this work was funded by the FFEU-ZWAP initiative of the Flemish Government.

\section{ABBREVIATIONS}

PGN, peptidoglycan; PFGSE, pulsed field gradient stimulated echo; STD, saturation transfer difference; PRE, paramagnetic relaxation enhancement; transferred NOE, transferred nuclear 
Overhauser enhancement; Ada, adamantylated glycine; D-iGln, D-isoglutamine; Lnk, linker residue

\section{REFERENCES}

(1) Harandi, A. M.; Medaglini, D.; Shattock, R. J.; Working Group convened by EUROPRISE. Vaccine adjuvants: A priority for vaccine research. Vaccine 2010, 28, 2363-2366.

(2) Oleszycka, E.; Lavelle, E. C. Immunomodulatory properties of the vaccine adjuvant alum. Curr. Opin. Immunol. 2014, 28, 1-5.

(3) Coffman, R. L.; Sher, A.; Seder, R. A. Vaccine adjuvants: putting innate immunity to work. Immunity 2010, 33, 492-503.

(4) Sorbara, M. T.; Philpott, D. J. Peptidoglycan: a critical activator of the mammalian immune system during infection and homeostasis. Immunol. Rev. 2011, 243, 40-60.

(5) Lise, L. D.; Audibert, F. Immunoadjuvants and analogs of immunomodulatory bacterial structures. Curr. Opin. Immunol. 1989, $2,269-274$.

(6) Myhre, A. E.; Aasen, A. O.; Thiemermann, C.; Wang, J. E. Peptidoglycan-an endotoxin in its own right? Shock 2006, 25, 227235.

(7) Szilagyi, L.; Pristovsek, P. Structural aspects of peptides with immunomodulating activity. Mini-Rev. Med. Chem. 2007, 7, 861-870.

(8) Ogawa, C.; Liu, Y.-J.; Kobayashi, K. Muramyl Dipeptide and its Derivatives: Peptide Adjuvant in Immunological Disorders and Cancer Therapy. Curr. Bioact. Compd. 2011, 7, 180-197.

(9) Girardin, S. E.; Boneca, I. G.; Viala, J.; Chamaillard, M.; Labigne, A.; Thomas, G.; Philpott, D. J.; Sansonetti, P. J. Nod2 Is a General Sensor of Peptidoglycan through Muramyl Dipeptide (MDP) Detection. J. Biol. Chem. 2003, 278, 8869-8872.

(10) Torchilin, V. P. Recent advances with liposomes as pharmaceutical carriers. Nat. Rev. Drug Discovery 2005, 4, 145-160.

(11) Sone, S.; Mutsuura, S.; Ogawara, M.; Tsubura, E. Potentiating effect of muramyl dipeptide and its lipophilic analog encapsulated in liposomes on tumor cell killing by human monocytes. J. Immunol. 1984, 132, 2105-2110.

(12) Effenberg, R.; Turánek Knötigová, P.; Zyka, D.; Čelechovská, H.; Mašek, J.; Bartheldyová, E.; Hubatka, F.; Koudelka, Š.; Lukáč, R.; Kovalová, A.; Šaman, D.; Křupka, M.; Barkocziova, L.; Kosztyu, P.; Šebela, M.; Drož, L.; Hučko, M.; Kanásová, M.; Miller, A. D.; Raška, M.; Ledvina, M.; Turánek, J. Nonpyrogenic Molecular Adjuvants Based on norAbu-Muramyldipeptide and norAbu-Glucosaminyl Muramyldipeptide: Synthesis, Molecular Mechanisms of Action, and Biological Activities in Vitro and in Vivo. J. Med. Chem. 2017, 60, $7745-7763$.

(13) Jakopin, Ž. Murabutide Revisited: A Review of its Pleiotropic Biological Effects. Curr. Med. Chem. 2013, 20, 2068.

(14) Ribić, R.; Habjanec, L.; Vranesić, B.; Frkanec, R.; Tomić, S. Synthesis and Biological Evaluation of New Mannose Derived Immunomodulating Adamantyltripeptides. Croat. Chem. Acta 2011, 84, 233-244.

(15) Ribić, R.; Habjanec, L.; Vranesić, B.; Frkanec, R.; Tomić, S. Synthesis and Immunostimulating Properties of Novel Adamant-1-yl Tripeptides. Chem. Biodiversity 2012, 9, 777-788.

(16) Ribić, R.; Habjanec, L.; Frkané́, R.; Vranesić, B.; Tomić, S. Influence of Mannosylation on Immunostimulating Activity of Adamant-1-yl Tripeptide. Chem. Biodiversity 2012, 9, 1373-1381.

(17) Davies, W. L.; Grunert, R. R.; Haff, R. F.; McGahen, J. W.; Neumayer, E. M.; Paulshock, M.; Watts, J. C.; Wood, T. R.; Hermann, E. C.; Hoffmann, C. E. Antiviral Activity of 1-Adamantanamine (Amantadine). Science 1964, 144, 862-863.

(18) Oxford, J. S.; Galbraith, A. Antiviral activity of amantadine: a review of laboratory and clinical data. Pharmacol. Therap. 1980, 11, $181-262$.

(19) Danielczyk, W. Twenty-five years of amantadine therapy in Parkinson's disease. J. Neural Transm., Suppl. 1995, 46, 399-405.

(20) Blanchet, P. J.; Metman, L. V.; Chase, T. N. Renaissance of amantadine in the treatment of Parkinson's disease. Adv. Neurol. 2003, 91, 251-257.
(21) Kilpatrick, G. J.; Tilbrook, G. S. Memantine. Merz. Curr. Opin. Invest. Drugs 2002, 3, 798-806.

(22) Francis, P. T. Glutamatergic Approaches to the Treatment of Cognitive and Behavioural Symptoms of Alzheimer's Disease. Neurodegener. Dis. 2008, 5, 241-243.

(23) Pinto, L. H.; Holsinger, L. J.; Lamb, R. A. Influenza virus M2 protein has ion channel activity. Cell 1992, 69, 517-528.

(24) Griffin, S. D.; Beales, L. P.; Clarke, D. S.; Worsfold, O.; Evans, S. D.; Jaeger, J.; Harris, M. P.; Rowlands, D. J. The p7 protein of hepatitis $\mathrm{C}$ virus forms an ion channel that is blocked by the antiviral drug, Amantadine. FEBS Lett. 2003, 535, 34-38.

(25) Lipton, S. A. Paradigm shift in neuroprotection by NMDA receptor blockade: memantine and beyond. Nat. Rev. Drug Discovery 2006, 5, 160-170.

(26) Jain, M. K.; Yen-Min Wu, N.; Morgan, T. K.; Briggs, M. S.; Murray, R. K. Phase transition in a lipid bilayer. II. Influence of adamantane derivatives. Chem. Phys. Lipids 1976, 17, 71-78.

(27) Phonphok, Y.; Rosenthal, K. S. Stabilization of clathrin coated vesicles by amantadine, tromantadine and other hydrophobic amines. FEBS Lett. 1991, 281, 188-190.

(28) Duff, K. C.; Cudmore, A. J.; Bradshaw, J. P. The location of amantadine hydrochloride and free base within phospholipid multilayers: a neutron and X-ray diffraction study. Biochim. Biophys. Acta, Biomembr. 1993, 1145, 149-156.

(29) Subczynski, W. K.; Wojas, J.; Pezeshk, V.; Pezeshk, A. Partitioning and Localization of Spin-Labeled Amantadine in Lipid Bilayers: An Epr Study. J. Pharm. Sci. 1998, 87, 1249-1254.

(30) Wang, J. F.; Schnell, J. R.; Chou, J. J. Amantadine partition and localization in phospholipid membrane: a solution NMR study. Biochem. Biophys. Res. Commun. 2004, 324, 212-217.

(31) White, K. L.; Rades, T.; Furneaux, R. H.; Tyler, P. C.; Hook, S. Mannosylated liposomes as antigen delivery vehicles for targeting to dendritic cells. J. Pharm. Pharmacol. 2006, 58, 729-737.

(32) Kerrigan, A. M.; Brown, G. D. C-type lectins and phagocytosis. Immunobiology 2009, 214, 562-575.

(33) Geijtenbeek, T. B. H.; Gringhuis, S. I. Signalling through Ctype lectin receptors: shaping immune responses. Nat. Rev. Immunol. 2009, 9, 465-479.

(34) Manček-Keber, M.; Ribić, R.; Chain, F.; Sinnaeve, D.; Martins, J. C.; Jerala, R.; Tomić, S.; Fehér, K. Adamantane Containing Peptidoglycan Fragments Enhance the Lipopolysaccharide-Induced Immune Response. Molecules, submitted for publication, 2020.

(35) Neuhaus, D.; Williamson, M. P. The Nuclear Overhauser Effect in Structural and Conformational Analysis; Wiley-VCH, 2000.

(36) Ni, F. Recent developments in transferred NOE methods. Prog Nucl. Magn. Reson. Spectrosc. 1994, 26, 517-606.

(37) Al-Abdul-Wahid, M. S.; Neale, C.; Pomes, R.; Prosser, R. S. A Solution NMR Approach to the Measurement of Amphiphile Immersion Depth and Orientation in Membrane Model Systems. J. Am. Chem. Soc. 2009, 131, 6452-6459.

(38) Hinton, D. P.; Johnson, C. S. Simultaneous measurement of vesicle diffusion coefficients and trapping efficiencies by means of diffusion ordered 2D NMR spectroscopy. Chem. Phys. Lipids 1994, $69,175-178$

(39) Morris, K. F.; Johnson, C. S. Diffusion-ordered two-dimensional nuclear magnetic resonance spectroscopy. J. Am. Chem. Soc. 1992, 114, 3139-3141.

(40) Mayer, M.; Meyer, B. Characterization of ligand binding by saturation transfer difference NMR spectroscopy. Angew. Chem., Int. Ed. 1999, 38, 1784-1788.

(41) Mayer, M.; Meyer, B. Group epitope mapping by saturation transfer difference NMR to identify segments of a ligand in direct contact with a protein receptor. J. Am. Chem. Soc. 2001, 123, 61086117.

(42) Angulo, J.; Nieto, P. M. STD-NMR: application to transient interactions between biomolecules-a quantitative approach. Eur. Biophys. J. 2011, 40, 1357-1369.

(43) Solomon, I. Relaxation Processes in a System of Two Spins. Phys. Rev. 1955, 99, 559-565. 
(44) Clore, G. M.; Iwahara, J. Theory, Practice, and Applications of Paramagnetic Relaxation Enhancement for the Characterization of Transient Low-Population States of Biological Macromolecules and Their Complexes. Chem. Rev. 2009, 109, 4108-4139.

(45) Nogueira, E.; Gomes, A. C.; Preto, A.; Cavaco-Paulo, A. Design of liposomal formulations for cell targeting. Colloids Surf., B 2015, 136, 514-526.

(46) Dürr, U. H. N.; Soong, R.; Ramamoorthy, A. When detergent meets bilayer: Birth and coming of age of lipid bicelles. Prog. Nucl. Magn. Reson. Spectrosc. 2013, 69, 1-22.

(47) Mäler, L.; Gräslund, A. Artificial Membrane Models for the Study of Macromolecular Delivery. In Macromolecular Drug Delivery; Belting, B., Ed.; Humana Press, 2009; Vol. 480, pp 129-139.

(48) Andersson, A.; Maler, L. Size and shape of fast-tumbling bicelles as determined by translational diffusion. Langmuir 2006, 22, 2447-2449.

(49) Ye, W.; Lind, J.; Eriksson, J.; Mäler, L. Characterization of the Morphology of Fast-Tumbling Bicelles with Varying Composition. Langmuir 2014, 30, 5488-5496.

(50) Beaugrand, M.; Arnold, A. A.; Hénin, J.; Warschawski, D. E.; Williamson, P. T. F.; Marcotte, I. Lipid Concentration and Molar Ratio Boundaries for the Use of Isotropic Bicelles. Langmuir 2014, 30, 6162-6170.

(51) Glover, K. J.; Whiles, J. A.; Wu, G. H.; Yu, N. J.; Deems, R.; Struppe, J. O.; Stark, R. E.; Komives, E. A.; Vold, R. R. Structural evaluation of phospholipid bicelles for solution-state studies of membrane-associated biomolecules. Biophys. J. 2001, 81, 2163-2171.

(52) Matsumori, N.; Murata, M. 3D structures of membraneassociated small molecules as determined in isotropic bicelles. Nat. Prod. Rep. 2010, 27, 1480-1492.

(53) Katsaras, J.; Harroun, T. A.; Pencer, J.; Nieh, M. P. "Bicellar" lipid mixtures as used in biochemical and biophysical studies. Naturwissenschaften 2005, 92, 355-366.

(54) Björnerås, J.; Nilsson, M.; Mäler, L. Analysing DHPC/DMPC bicelles by diffusion NMR and multivariate decomposition. Biochim. Biophys. Acta, Biomembr. 2015, 1848, 2910-2917.

(55) Sklenar, V.; Piotto, M.; Leppik, R.; Saudek, V. GradientTailored Water Suppression for 1H-15N HSQC Experiments Optimized to Retain Full Sensitivity. J. Magn. Reson., Ser. A 1993, 102, 241-245.

(56) Sinnaeve, D. The Stejskal-Tanner equation generalized for any gradient shape-an overview of most pulse sequences measuring free diffusion. Concepts Magn. Reson., Part A 2012, 40A, 39-65.

(57) Vranken, W. F.; Boucher, W.; Stevens, T. J.; Fogh, R. H.; Pajon, A.; Llinas, P.; Ulrich, E. L.; Markley, J. L.; Ionides, J.; Laue, E. D. The CCPN data model for NMR spectroscopy: Development of a software pipeline. Proteins: Struct., Funct., Bioinf. 2005, 59, 687-696.

(58) Brünger, A. T.; Adams, P. D.; Clore, G. M.; DeLano, W. L.; Gros, P.; Grosse-Kunstleve, R. W.; Jiang, J. S.; Kuszewski, J.; Nilges, M.; Pannu, N. S.; Read, R. J.; Rice, L. M.; Simonson, T.; Warren, G. L. Crystallography \& NMR system: A new software suite for macromolecular structure determination. Acta Crystallogr., Sect. D: Biol. Crystallogr. 1998, 54, 905-921.

(59) Linge, J. P.; Williams, M. A.; Spronk, C.; Bonvin, A.; Nilges, M. Refinement of protein structures in explicit solvent. Proteins: Struct., Funct., Bioinf. 2003, 50, 496-506.

(60) Discovery Studio Visualiser, version 3.5; Accelrys, 2012.

(61) The PyMOL Molecular Graphics System, version 1.2; Schrödinger, LLC, 2014.

(62) Yan, J. L.; Kline, A. D.; Mo, H. P.; Shapiro, M. J.; Zartler, E. R. The effect of relaxation on the epitope mapping by saturation transfer difference NMR. J. Magn. Reson. 2003, 163, 270-276.

(63) Kemper, S.; Patel, M. K.; Errey, J. C.; Davis, B. G.; Jones, J. A.; Claridge, T. D. W. Group epitope mapping considering relaxation of the ligand (GEM-CRL): Including longitudinal relaxation rates in the analysis of saturation transfer difference (STD) experiments. J. Magn. Reson. 2010, 203, 1-10.

(64) Stott, K.; Stonehouse, J.; Keeler, J.; Hwang, T.-L.; Shaka, A. J. Excitation Sculpting in High-Resolution Nuclear Magnetic Resonance
Spectroscopy: Application to Selective NOE Experiments. J. Am. Chem. Soc. 1995, 117, 4199-4200.

(65) Aguilar, J. A.; Nilsson, M.; Bodenhausen, G.; Morris, G. A. Spin echo NMR spectra without J modulation. Chem. Commun. 2012, 48, 811-813.

(66) Andersson, A.; Maler, L. Motilin-bicelle interactions: membrane position and translational diffusion. FEBS Lett. 2003, 545, 139-143.

(67) Chou, J. J.; Baber, J. L.; Bax, A. Characterization of phospholipid mixed micelles by translational diffusion. J. Biomol. NMR 2004, 29, 299-308.

(68) Andersson, A.; Maler, L. Magnetic resonance investigations of lipid motion in isotropic bicelles. Langmuir 2005, 21, 7702-7709.

(69) Lind, J.; Nordin, J.; Maler, L. Lipid dynamics in fast-tumbling bicelles with varying bilayer thickness: Effect of model transmembrane peptides. Biochim. Biophys. Acta, Biomembr. 2008, 1778, 2526-2534.

(70) Ye, W.; Liebau, J.; Mäler, L. New Membrane Mimetics with Galactolipids: Lipid Properties in Fast-Tumbling Bicelles. J. Phys. Chem. B 2013, 117, 1044-1050.

(71) Unnerståle, S.; Mäler, L. pH-Dependent Interaction between C-Peptide and Phospholipid Bicelles. J. Biophys. 2012, 2012, No. 185907.

(72) Geudens, N. Insights in the Structure-Function and Membrane Interactions of Natural Cyclic Lipodepsipeptides, PhD Thesis, 2017.

(73) Andersson, A.; Almqvist, J.; Hagn, F.; Mäler, L. Diffusion and dynamics of penetratin in different membrane mimicking media. Biochim. Biophys. Acta, Biomembr. 2004, 1661, 18-25.

(74) Halassy Spoljar, B.; Čimbora, T.; Hanzl-Dujmović, I.; Dojnović, B.; Sabioncello, A.; Krstanović, M.; Tomašić, J. Influence of adjuvantactive peptidoglycan monomer on specific $\mathrm{T}$ cell responses in mice. Vaccine 2002, 20, 3543-3550.

(75) Frkanec, R.; Travas, D.; Krstanovic, M.; Spoljar, B. H.; Ljevakovic, D.; Vranesic, B.; Frkanec, L.; Tomasic, J. Entrapment of peptidoglycans and adamantyltripeptides into liposomes: An HPLC assay for determination of encapsulation efficiency. J. Liposome Res. 2003, 13, 279-294.

(76) Angulo, J.; Enríquez-Navas, P. M.; Nieto, P. M. LigandReceptor Binding Affinities from Saturation Transfer Difference (STD) NMR Spectroscopy: The Binding Isotherm of STD Initial Growth Rates. Chem. - Eur. J. 2010, 16, 7803-7812.

(77) Quirós, M. T.; Angulo, J.; Muñoz, M. P. Kinetics of intramolecular chemical exchange by initial growth rates of spin saturation transfer difference experiments (SSTD NMR). Chem. Commun. 2015, 51, 10222-10225.

(78) Štimac, A.; Segota, S.; Sikirić, M. D.; Ribić, R.; Frkanec, L.; Svetlicić, V.; Tomić, S.; Vranešić, B.; Frkanec, R. Surface modified liposomes by mannosylated conjugates anchored via the adamantyl moiety in the lipid bilayer. Biochim. Biophys. Acta, Biomembr. 2012, $1818,2252-2259$.

(79) Wijagkanalan, W.; Kawakami, S.; Takenaga, M.; Igarashi, R.; Yamashita, F.; Hashida, M. Efficient targeting to alveolar macrophages by intratracheal administration of mannosylated liposomes in rats. J. Controlled Release 2008, 125, 121-130.

(80) Allavena, P.; Chieppa, M.; Monti, P.; Piemonti, L. From pattern recognition receptor to regulator of homeostasis: The double-faced macrophage mannose receptor. Crit. Rev. Immunol. 2004, 24, 179192.

(81) Over, B.; McCarren, P.; Artursson, P.; Foley, M.; Giordanetto, F.; Gronberg, G.; Hilgendorf, C.; Lee, M. D.; Matsson, P.; Muncipinto, G.; Pellisson, M.; Perry, M. W. D.; Svensson, R.; Duvall, J. R.; Kihlberg, J. Impact of Stereospecific Intramolecular Hydrogen Bonding on Cell Permeability and Physicochemical Properties. J. Med. Chem. 2014, 57, 2746-2754.

(82) Wang, X.; Ramström, O.; Yan, M. Dynamic light scattering as an efficient tool to study glyconanoparticle-lectin interactions. Analyst 2011, 136, 4174-4178.

(83) Ribić, R.; Paurević, M.; Tomić, S. Advances in Desmuramyl Peptide Research. Croat. Chem. Acta 2019, 92, 153. 